\title{
Assessment of an All-Particle Hybrid Method for Hypersonic Rarefied Flow
}

\author{
Eunji Jun* and Iain D. Boyd $^{\dagger}$ \\ Department of Aerospace Engineering, University of Michigan, Ann Arbor, MI 48109
}

\begin{abstract}
Hypersonic aerothermodynamics for a probe entering a planetary atmosphere is an important issue in space exploration. The probe experiences various Knudsen number regimes, ranging from rarefied to continuum, due to density variation in the planet's atmosphere. To simulate such multiscale flows, a novel hybrid particle scheme is employed in the present work. The hybrid particle scheme employs the direct simulation Monte Carlo (DSMC) method in rarefied flow regions and the low diffusion (LD) particle method in continuum flow regions. Numerical procedures in the low diffusion particle method are implemented within an existing DSMC algorithm. The hybrid scheme is assessed by studying Mach 10 nitrogen flow over a sphere with a global Knudsen number of 0.002. Standard DSMC and CFD results are compared with the LD-DSMC hybrid simulation results. The hybrid scheme results show good overall agreement with results from standard DSMC computation, while CFD is inaccurate especially in the wake where a highly rarefied region exists. The LD-DSMC hybrid solution is able to increase computational efficiency by $20 \%$ in comparison to DSMC. Also, a module initializing the LD-DSMC hybrid method with a Navier-Stokes solution is developed. The initialized solution agrees well with DSMC and requires only $45 \%$ of the resources.
\end{abstract}

\section{Nomenclature}

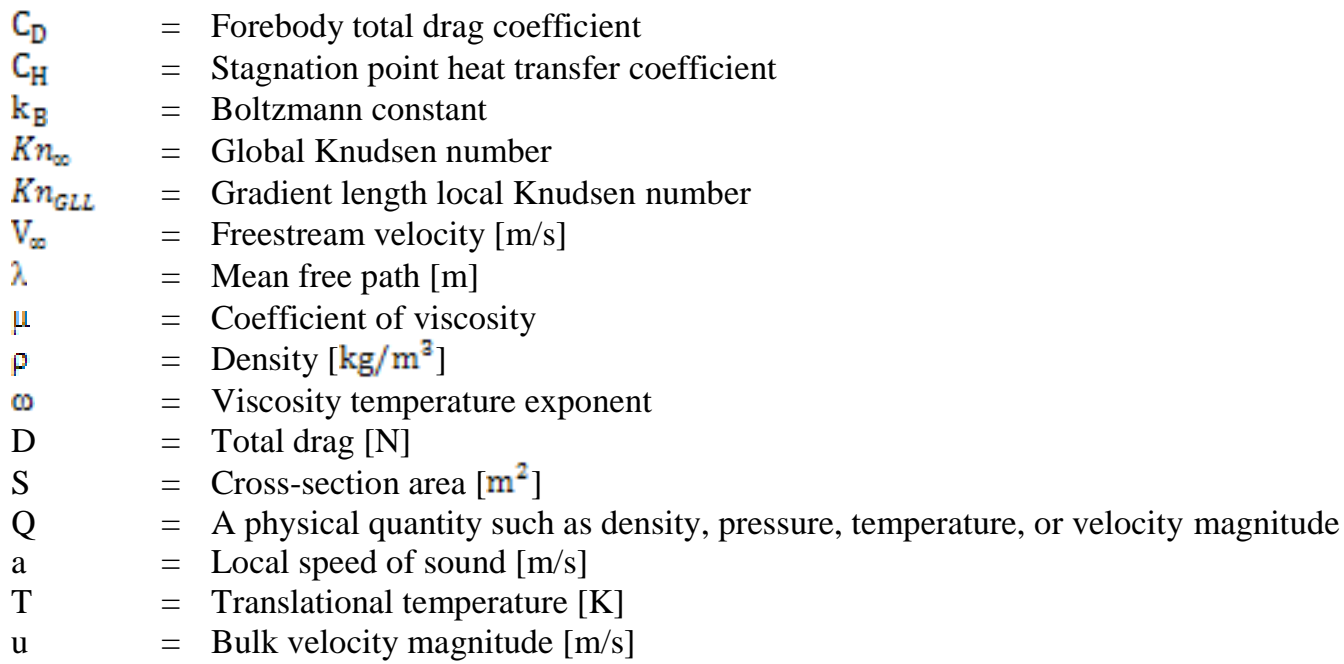

\section{Introduction}

$\mathrm{T}$ HE desire of humans for space exploration was first realized through the success of Sputnik in 1957 and over 200 missions have been completed or are planned since that time. ${ }^{1}$ As we move into the 21 st century, planetary missions, especially to the Moon and Mars, have taken center stage in planetary exploration due to the possibility of finding resources and life. ${ }^{2}$ The most recent planetary mission is the Mars Science Laboratory (MSL), which is a robotic mission to Mars launched by NASA on November 26, 2011 that successfully landed Curiosity, a Mars rover,

\footnotetext{
* Post-doctoral Research Fellow, Member AIAA

${ }^{\dagger}$ James E.Knott Professor, Fellow AIAA
} 
on August 6, 2012. ${ }^{1}$ Planetary missions are classified by type of approach to the destination, like orbiters, flybys, hard impactors and landers. The first successful soft landing mission was Luna 9 in 1966; many lander missions have been performed since then. ${ }^{3}$ One of the most challenging parts of the landing missions is the entry, descent, and landing (EDL) sequence.

To explore a planet possessing an atmosphere, most planetary missions utilize an entry vehicle. As a vehicle enters the atmosphere of a planet, a series of physical interactions ensue between the vehicle and the surrounding atmospheric gas. The vehicle enters the atmosphere at near-orbital velocity relative to the atmosphere. The velocity is much higher than the speed of sound in the atmosphere; hypersonic Mach numbers greater than 20 are encountered. At the high Mach numbers, a strong bow shock wave is located close to the vehicle surface. The total energy crossing the bow shock of the vehicle is sufficiently large to excite internal vibrational energy, and to cause dissociation and even ionization. These high temperatures change the gas properties, so it is a dominant effect of hypersonic aerodynamics. ${ }^{4}$ For example, the Mars Pathfinder (MPF) entry vehicle entered the Mars atmosphere directly from the interplanetary transfer trajectory with an inertial velocity magnitude of $7,460 \mathrm{~m} / \mathrm{s}$ at $140 \mathrm{~km}$ above the surface. As the vehicle descended deeper into the atmosphere, it experienced an extreme flow condition. The vehicle reached $56 \mathrm{~km}$ altitude within $50 \mathrm{sec}$. The maximum heat flux at stagnation point was $20 \mathrm{~W} / \mathrm{cm}^{2}$ before the deployment of a parachute. 5,6

In the EDL sequence, the flow experiences a wide range of regimes including both high and low Knudsen number. The flow regime is classified as free molecular flow, transitional flow and continuum flow depending on the Knudsen number. ${ }^{7}$ Some flow regions are within the low Knudsen number range for which the Euler or NavierStokes equations are valid, while other regions are characterized by strong thermal non-equilibrium and require a simulation method based on kinetic theory. Even when an entry vehicle as a whole is in the continuum regime, there may exist locally rarefied regions in the hypersonic flowfield such as interior of shock waves, wall boundary layers or wake regions. In these regions, the numbers of particles and collisions may not be enough to produce a Maxwellian distribution and an equilibrium description is inaccurate. ${ }^{8}$ This kind of mixed flow is called a multiscale flow. Thus, a multiscale flow that experiences a wide range of regimes includes both high and low Knudsen number. Figure 1 is an example of a multiscale flow. The contours in Figure 1 are of the maximum gradient length local Knudsen number. The global Knudsen number and the Mach number of the freestream are 0.002 and 10 , respectively, and the freestream condition is summarized in Table 1. The flow contains a wide range of Knudsen number and it might be classified as continuum or non-continuum region based on the gradient length local Knudsen number. In these types of flows, a near-equilibrium gas velocity distribution may exist through much of the flowfield, as the equilibrating effect of intermolecular collisions dominates over other processes, such as inhomogeneous diffusive transport of gas-surface interaction, which tend to pull the velocity distribution away from equilibrium. However, some flowfield regions may have a characteristic length scale comparable to or smaller than the local mean free path, so that the influence of collisions does not dominate and the velocity distribution diverges considerably from the equilibrium limit. Many flows include both high Knudsen number and low Knudsen number regions; typical examples are high speed flow (atmospheric flow around reentry vehicles, other hypersonic aircrafts), and low density flow (rocket exhaust plumes at high altitude). The Euler or Navier-Stokes equations are valid for the low Knudsen number regime flow, and CFD simulation is performed to solve those equations numerically. On the other hand, the Boltzmann equation (gas kinetic theory) is valid everywhere in both low Knudsen number and high Knudsen number regimes and the velocity distribution is determined by numerically solving the Boltzmann equation or applying a Monte Carlo method. ${ }^{8}$ However, these methods are very expensive to apply in the low Knudsen number regime. Therefore, it is attractive to develop a hybrid method to analyze multiscale flow.

Development to simulate multiscale flow which involves a wide range of Knudsen number regimes includes "all-particle" hybrid schemes." ${ }^{10}$ Hybrid simulation approaches have been proposed using DSMC type particles throughout the simulation domain in which equilibrium particle methods are used to simulate continuum flow regions. ${ }^{9,11-13}$ This equilibrium particle method uses particles to transport mass, momentum and energy through a computational grid, and employs temporal decoupling between particle movement and velocity re-sampling procedures during each simulation time step. For the whole domain, macroscopic flow properties are calculated in both rarefied and continuum regions by averaging quantities among representative particles. Particle velocities are redistributed under an assumption of local equilibrium for continuum regions. This method can have very strong coupling between the two flow regimes, also, it has simple code development, information transfer, and no need to integrate two very different simulation schemes in the same code. However, these "all-particle" hybrid techniques are prone to large errors associated with numerical diffusion resulting from free-molecular fluxes between adjacent cells. ${ }^{10,14,15}$ This numerical diffusion is due to an extreme sensitivity to computational cell size in the equilibrium particle method. Random molecular motion in the continuum flows is almost suppressed on length scales comparable to the cell size because the ratio of local mean free path to cell size typically approaches zero. Also the 
individual molecules tend to move along smooth trajectories which approximately follow the gas streamlines on these macroscopic scales. In contrast, representative particles in the equilibrium particle method exhibit random motion on length scales comparable to the cell size, so that the cell size takes on properties of the local mean free path and becomes a scaling factor for numerical transport coefficients. Because the influence of collisions is neglected in fluxes based on particle motion between adjacent cells, the ratio of numerical transport coefficients (viscosity, thermal conductivity and mass diffusivity) to physical transport coefficients tends to scale with the ratio of the mean free path to the cell size. So, both numerical viscosity and thermal conductivity become extremely large when the cell size is much greater that the local mean free path.

In this study, a different equilibrium particle method ${ }^{16-19}$ is used as an alternative means of computing continuum gas flows regions in a hybrid simulation. In this method, intended for simulating gas flows near the low Knudsen number limit, a large number of representative particles are tracked through the grid in such a way that every particle maintains a constant relative position within a network of Lagrangian cells. Each Lagrangian cell is coincident with a cell in the fixed Eulerian grid at the beginning of each time step, and moves and deforms over the time step interval according to local bulk gas properties. Particles follow the macroscopic motion of Lagrangian cells, and move along trajectories which closely approximate the gas streamlines. Random particle motion associated with thermal energy is therefore greatly suppressed. As a result, numerical diffusion errors, as well as effects of statistical scatter, are generally far smaller than in other equilibrium particle methods. The method presented here improves existing equilibrium particle methods by significantly reducing both numerical diffusion effects and statistical scatter, so it is termed as the low diffusion (LD) method.

This paper will discuss numerical procedures in the LD method, the LD-DSMC hybrid method and the LDDSMC initialization with a Navier-Stokes solution. A Mach 10 flow of nitrogen over a 12 inch diameter sphere is considered to assess the validity of the LD-DSMC hybrid scheme. Simulations are performed with standard DSMC, CFD and the LD-DSMC hybrid method. The LD-DSMC hybrid results for flowfield and surface properties are compared with standard DSMC as well as the CFD solution. The computational efficiency of the hybrid method for this flow is presented and discussed. Then, LD-DSMC initialized with a Navier-Stokes solution is evaluated for the same hypersonic flow over the sphere. The results are compared with the conventional LD-DSMC hybrid method, and computational efficiency is again discussed. Finally, the paper concludes with a summary.

\section{A. The Low Diffusion Particle Method}

\section{Numerical Method and Physical Models}

A particle technique, termed the low diffusion (LD) method, to describe equilibrium gas was developed by Burt et al. ${ }^{17,20,21}$ The numerical procedures in the LD particle simulations are similar to the DSMC technique. Both LD and DSMC divide the computational domain into a large number of grid cells, and representative particles are tracked through the cells. As in DSMC, all particles in an LD simulation carry information of position, a velocity used to update the position, and a species identification number. However, in a LD method simulation each particle is also assigned a temperature and a second velocity, termed the 'bulk particle velocity', which is used to allocate momentum among all particles in each cell. One main difference between LD and DSMC is in dealing with the particle collisions. The LD particles undergo an alternative procedure in place of DSMC particle collisions. The alternate collision procedures enforce an equilibrium velocity distribution in each cell.

The LD method simulates the flow as a collection of continuous, finite volume, and equilibrium reservoirs, represented by Lagrangian cells. The Lagrangian cells correspond to the Lagrangian flow description, which is a way of looking at fluid motion where the observer follows an individual fluid particle as it moves through space and time. Every particle remains fixed with respect to the assigned Lagrangian cell over the time step interval. Each Lagrangian cell is coincident with a cell in a fixed Eulerian grid at the beginning of each time step, and moves and deforms over the time step interval. Particles follow the macroscopic motion of Lagrangian cells, and move along trajectories which closely approximate the gas streamlines.

During each time step, particles are moved through the grid according to their assigned velocities and bulk flow properties are calculated by averaging quantities over all particles in each cell. Another main difference between the LD method and DSMC is related to how the velocities used for particle movement are updated during each time step. The LD method starts from the calculation of cell bulk velocity, mass density and the thermal speed for each cell. These values are evaluated based on cell-averaged particle values, and are stored in the cell data structure. Once these quantities have been determined for all cells during the current time step, a series of Lagrangian face velocities are computed. Lagrangian cells are superimposed over fixed Eulerian cells, with identical face locations at the beginning of the timestep. No net momentum or energy can be transferred to the gas from a Lagrangian cell face, but both momentum and energy may be exchanged through a face between two neighboring cells. To account for momentum and energy exchange through Lagrangian faces, bulk velocity and temperature values are updated in 
each cell during each time step. Then, all particles in each cell are assigned the cell-based bulk velocity and temperature. Finally, the position of each Lagrangian cell vertex at the end of the time step interval is calculated and the velocity used for particle movement is updated. Numerical procedures in the LD method are described in detail in Ref. 22.

\section{B. LD-DSMC Hybrid Numerical Method for Multiscale Flow}

For simulation of a multiscale flow that has mixed equilibrium and non-equilibrium regions, a hybrid method provides a numerically efficient approach. The LD-DSMC hybrid method employs the DSMC method in the nonequilibrium region and the $\mathrm{LD}$ method in the equilibrium region.

To employ both LD and DSMC methods as a part of the hybrid scheme, it is necessary to determine continuum breakdown to allocate cells to LD and DSMC domains. Boyd et al. proposed the use of the gradient length local (GLL) Knudsen number. ${ }^{22,23}$

$$
\mathrm{Kn}_{\mathrm{GLL}}=\frac{\mathrm{\lambda}}{\mathrm{Q}}\left|\frac{\mathrm{dQ}}{\mathrm{dl}}\right|
$$

where $\lambda$ is the mean free path, 1 is some distance between two points in the flow field and Q is some flow quantity of interest, such as density, pressure, temperature, or velocity magnitude. In this work, LD-DSMC domain decomposition is determined based on the following GLL Knudsen number:

$$
\mathrm{Kn}_{\mathrm{GLL}, \max }=\max \left\langle\frac{\lambda}{\rho}|\nabla \rho|,\left.\frac{\lambda}{\mathrm{T}}|\nabla \mathrm{T}|\right|_{\mathrm{a}} ^{\frac{\lambda}{2}}|\nabla \mathrm{u}|\right\rangle
$$

where $\lambda$ is again the mean free path, $p$ is the density, $T$ is the translational temperature, $u$ is the bulk velocity magnitude, and a is the local speed of sound.

To hybridize these two methods, region allocation and information transfer between regions are essential. In addition, the LD method must employ physical models that are consistent with DSMC to reduce information loss across continuum breakdown boundaries. Regions for which $\mathrm{Kn}_{\mathrm{GLL} \max }$ is larger than 0.05 are assigned to the DSMC/non-equilibrium domain, and all other regions are assigned to LD/equilibrium domain. Domain decomposition is performed once every few thousand time steps. After assigning the LD and DSMC domains, information must be transferred across the domain boundaries. Two layers of overlapping cells are employed along the boundary between DSMC and LD domains, and are designated as buffer regions A and B. These buffer regions are used for information exchange between the DSMC and LD methods. Each buffer region is positioned along the boundary between DSMC and LD domains, such that a line drawn from a DSMC cell to a nearby LD cell will pass through at least two cells in buffer region A, and then through at least two cells in region B. The relative locations of these buffer regions are illustrated in Figure 2.

The LD method must have physical models that are consistent with DSMC to reduce information loss across the continuum breakdown boundaries. The LD method for inviscid flow cannot properly resolve boundary layers of other regions involving large transverse gradients because the equilibrium assumption underlying the LD method makes it incapable of modeling physically realistic diffusive transport. To model viscous flow effects, the diffusive term in the Navier-Stokes equations are evaluated in each cell during each time step. ${ }^{16}$ The diffusive term is used to modify particle velocity and temperature values in a manner consistent with DSMC transport coefficients at the near equilibrium limit. To account for viscous flow effects, diffusive momentum and energy transport as well as momentum and energy transfer across the Lagrangian cell faces are accounted for in the reassignment of particle bulk velocity and temperature.

Thermal non-equilibrium effects may become significant at high temperature and high Knudsen number. So, rotational and vibrational non-equilibrium models need to be included in the continuum region when significant internal energy non-equilibrium effects exist. This improves consistency with the DSMC method and reduces information loss across continuum breakdown boundaries. ${ }^{24}$

For accurate simulation, the standard DSMC method requires small enough computational cells, a small enough time step, and a large enough number of particles per cell. ${ }^{8}$ The DSMC cell size should be smaller than the mean free path, and the DSMC time step interval should be smaller than the mean collision time, to obtain valid results. However, the LD method is relatively independent of such requirements. So, LD cells can be larger compared with DSMC cells and the relatively larger LD cells may increase the efficiency of the LD-DSMC hybrid simulation. Hybrid simulations are performed on a mesh which is sufficiently coarse for mesh independence in LD calculations, but coarser than necessary to meet DSMC guidelines. The DSMC cells are, therefore, divided into subcells with

4

American Institute of Aeronautics and Astronautics 
subcell dimensions set to approximately half the mean free path. Collision partners are then selected from particles located in either the same subcell or a neighboring subcell. This procedure can reduce the mean collision separation between two molecules that are selected as collision partners. ${ }^{21}$ In the current LD-DSMC algorithm, a DSMC cell is divided into subcells if it does not satisfy the DSMC requirement and each subcell is forced to have a certain number of particles. Otherwise, all other cells have the 'desired number of particles'. This promotes a uniform and sufficient number of particles per cell. In this research, the desired number of particles per cell is 30 , the maximum number of subcells in each direction is 20, and the desired number of particles per subcell is 4 .

Although the subcell utility is used to satisfy the DSMC guidelines on a much coarser mesh, larger cells may induce too many particles per cell that may decrease computation efficiency. To control this, an adaptive numerical weight scale factor which determines the number of particles per cell is used. In the DSMC or LD simulation, many real particles are treated as one simulation particle. The numerical weight is a parameter used to represent how many real particles are treated as one simulation particle. Thus, a cell with a weight scale factor of 0.5 will have a particle weight of one half the reference values. The weight scale factor is declared in each cell of the domain.

\section{Initialization with a Navier-Stokes Solution}

A more common hybrid method for multiscale flow is the CFD-DSMC hybrid simulation which starts its calculation from an initial CFD solution that provides the starting domain decomposition. By comparison, the LDDSMC hybrid approach currently relies on a very expensive initial DSMC simulation to provide prediction of continuum breakdown. This means the whole computational domain is simulated by standard expensive DSMC until a first continuum breakdown can be evaluated. For the hypersonic flow studied here, the pure LD method is initially numerically unstable, so it does not provide a reliable way to initialize the computational domain. When multiscale flow is simulated only with the DSMC method, the continuum regions will have many particles and collisions per cell. This is computationally expensive. Generally, several thousand steps are required until a first continuum breakdown evaluation can be performed. After initial continuum breakdown, the LD method is used for continuum cells and DSMC is used elsewhere. A more efficient way to operate the LD-DSMC hybrid method is to use the LD method in continuum regions from the startup of the simulation. To accomplish this, an initial domain decomposition is needed and a CFD simulation is used to obtain it. CFD is not an adequate method to analyze multiscale flow which includes both equilibrium and non-equilibrium flow regions. However, it may provide an approximate initial domain decomposition.

From the CFD solution, the domain is decomposed based on the gradient length local Knudsen number, $\mathrm{Kn}_{\mathrm{GLL} \max }$. The local mean free path is calculated using the macroscopic properties in each cell as:

$$
\lambda=\frac{2(5-2 \omega)(7-2 \omega)}{15} \sqrt{\frac{\mathrm{m}}{2 \pi \mathrm{k}_{\mathrm{B}} \mathrm{T}}\left(\frac{\mu}{\rho}\right)}
$$

where $\mu$ is the local viscosity, $\omega$ is the viscosity temperature exponent, $\mathrm{k}_{\mathrm{B}}$ is the Boltzmann constant. 0.05 is the reference value of $\mathrm{Kn}_{\text {GLL } \max }$ used to classify continuum or non-continuum cells. Regions for which $\mathrm{Kn}_{\text {GLL max }}$ is larger than 0.05 are assigned as DSMC domains, and all other regions are assigned as LD domains. In the DSMC domain, a conventional CFD cell may be too large to satisfy the DSMC guideline that the cell size should be smaller than the mean free path. After LD/DSMC domain decomposition based on the CFD solution, therefore, the DSMC cells may need to be refined.

After LD/DSMC domain decomposition, every quadrilateral CFD cell is classified as a DSMC or LD cell. Within the DSMC domains, the non-continuum cell-centered value of mean free path, $\lambda$, is compared to the length of each cell face in order to determine the refinement required in each cell of the initial CFD mesh. The mesh refinement restricts the cell size to $\lambda$ in the direction of the flow gradient while $4 \lambda$ is allowed in the direction normal to the flow gradient. The refinement factor, $\mathrm{r}_{\mathrm{f}}$, of each cell face is calculated as

$$
\mathrm{r}_{\mathrm{f}}=\left\lfloor\mathrm{l}_{\mathrm{f}} \times\left[\mathrm{F} \lambda\left(3\left|\hat{\mathrm{n}}_{\mathrm{f}} \times \frac{\nabla \mathrm{Q}}{\|\nabla \mathrm{Q}\|}\right|+1\right)\right]^{-1}\right\rfloor
$$

where $l_{\mathrm{f}}$ is the length of the cell face, $\frac{\mathbb{Q Q}}{|\mathrm{QQ}|}$ is the unit gradient vector, and the dot product determines the alignment of the cell face with the direction of the gradient. For consistency with continuum breakdown, $\mathrm{Q}$ is calculated with the same flow quantities $p, T_{s}$ or u, which are used for domain decomposition in Equation (2). F is a scaling factor used as a multiple of the local mean free path, $\lambda$. \」 $\$ rounds to the nearest integer. Each non-continuum quadrilateral cell has two refinement factors, $r_{1}, r_{2}$ and the opposite faces should have the same refinement factor. Due to the fact that each cell face borders with another cell, the refinement factors must be consistent with the refinement in each neighboring cell. Figure 3-(a) shows a conceptual procedure of cell refinement. From the initial CFD structured grid, 
the cells are classified as continuum (LD) or non-continuum (DSMC) cells. Then, the DSMC cells are refined to satisfy DSMC guidelines. LD cells are left without refinement. After the cell refinement in the DSMC cells, connection cells are needed to connect to the LD cells. The connection cells consist of triangular cells, and their number depends on the refinement factor of the boarder face of the DSMC cell. The connection cell may have from 1 to 3 border faces. If the connection cell has 4 border faces, it automatically belongs to a DSMC region. Figure 3(b) shows the refined mesh for a LD-DSMC hybrid simulation. The region near the bow shock belongs to the noncontinuum region, and it is refined to small cells while continuum regions are left as the initial LD cells. The initial cell refinement is based on the Navier-Stokes solution that cannot give an exact solution for the non-equilibrium flow. So, during the hybrid LD-DSMC simulation, domain decomposition is re-evaluated periodically until a steady state is reached.

\section{Simulation Cases}

Nitrogen flow over a 12 inch diameter sphere at Mach 10 is studied. The free stream temperature is $200 \mathrm{~K}$ giving a free stream velocity of $2880 \mathrm{~m} / \mathrm{s}$. The surface of the sphere has a fixed temperature of $500 \mathrm{~K}$. The mass density, number density and mean free path are given in Table 1. The Knudsen number is calculated using the sphere diameter as the characteristic length and the hard sphere model is used to determine the mean free path. For both standard DSMC and the LD-DSMC simulations, the same physical models are employed. Molecular collisions are simulated using the variable hard sphere (VHS) model with a reference temperature of $273 \mathrm{~K}$ and a viscosity temperature exponent of 0.75 . The no-time-counter (NTC) scheme of Bird is used to select DSMC collision pairs during each time step. ${ }^{8}$ Translational-rotational energy exchange during simulated DSMC collisions is determined using the Larsen Borgnakke statistical model along with the total collision energy model of Boyd ${ }^{25}$ The average probability of an inelastic collision in which vibrational energy is exchanged with translational energy is determined using a variable probability with correlated experimental data from Millikan and White. ${ }^{26}$ The current solutions are obtained without chemical reactions.

Table 1. Simulation flow properties for $\mathrm{N}_{2}$ hypersonic flow over a sphere

\begin{tabular}{cccc}
\hline $\mathrm{Kn}_{\mathrm{ws}}$ & $\begin{array}{c}\text { Mass Density } \\
{\left[\mathrm{kg} / \mathbf{m}^{3}\right]}\end{array}$ & $\begin{array}{c}\text { Number Density } \\
{\left[\text { particles } / \mathbf{m}^{3}\right]}\end{array}$ & $\begin{array}{c}\text { Mean Free Path } \\
{[\mathrm{m}]}\end{array}$ \\
\hline $\mathbf{0 . 0 0 2}$ & $9.88 \times 10^{-5}$ & $2.21 \times 10^{21}$ & $6.10 \times 10^{-4}$ \\
\hline
\end{tabular}

A hybrid mesh is used in which a structured grid is employed along the fore body surface while an unstructured grid is used elsewhere in the flow field (Figure 4). For the structured grid, cell stretching is employed to provide enough particles per cell near the stagnation point. This is useful technique in axisymmetric simulations, where it is often difficult to achieve a sufficiently large number of particles per cell near the axis.

\section{A. Comparing DSMC, LD-DSMC, and CFD}

\section{Result and Discussion}

Standard DSMC, LD-DSMC, and CFD calculations are performed. Flowfield contours and surface properties are used to evaluate the LD-DSMC method. Profiles of flow properties along extracted lines and wake rarefaction effects are also discussed. The simulation cases are summarized in Table 1. Standard DSMC simulation is performed using the DSMC code MONACO, and the LD-DSMC hybrid simulation is performed using a modified version of MONACO. ${ }^{27}$ The Navier-Stokes equations are solved using the Michigan Aerothermodynamics NavierStokes (LeMANS) solver. ${ }^{28}$ LeMANS is able to simulate 2D, axi-symmetric and 3D flows on parallel computing architectures. Time integration of the governing equations uses a point-implicit method. A wall slip boundary condition is employed in the CFD calculation. ${ }^{29}$

At LD-DSMC simulation startup, the entire flow field is assigned to the DSMC domain. The continuum breakdown parameter based on density, temperature and velocity gradients is then periodically evaluated in all cells within the computational grid. When the breakdown parameter value is larger than 0.05 in a given cell, continuum breakdown is assumed and the cell is assigned to the DSMC domain (Table 2).

Figure 5 shows continuum breakdown domain boundaries based on the maximum gradient length local Knudsen number, $\mathrm{Kn}_{\text {GLL max }}$. The upper half is from DSMC and the lower half is from the LD-DSMC hybrid simulation. The LD domain includes near-equilibrium regions in the freestream and past-shock regions, whereas the DSMC domain comprises the remaining portions of the bow shock and wake where a high degree of non-equilibrium is observed.

Figure 6 shows the velocity contours from DSMC, LD-DSMC hybrid, and CFD. Streamlines show generally expected trends. The flow separates from the rear surface, and a wake forms. In Figure 6-(a), the upper half is 
simulated by the standard DSMC scheme and the low half is simulated by the LD-DSMC hybrid method. Very good agreement is observed between standard DSMC and the LD-DSMC hybrid results. For example, the shock standoff distance and shock shapes are nearly identical in the solutions. In the hybrid method, the detached bow shock is simulated in the DSMC domain, and the boundary layer near the sphere surface is also assigned to DSMC. The flow in the wake of a hypersonic blunt body involves highly rarefied conditions, so this region is also assigned as a DSMC domain. The freestream and high density post shock regions are assigned to the LD domain. In Figure 6-(b), the lower half is simulated by the LD-DSMC hybrid method and the upper half is simulated by CFD with slip boundary condition. Generally, since DSMC is a particle method with accuracy in both the continuum and the rarefied regimes, it is assumed in this study that the DSMC results are the most accurate.

Table 2. Simulation cases for $\mathrm{N}_{2}$ hypersonic flow over a sphere

\begin{tabular}{c|c|c|c}
\hline Cases & Total cells & \multicolumn{2}{|c}{ Representative method according to the regimes } \\
\cline { 3 - 4 } & & Continuum & Non-continuum \\
\hline Standard DSMC & $1,071,858$ & DSMC & DSMC \\
\hline CFD, slip & 100,000 & CFD & CFD \\
\hline LD-DSMC & 234,256 & LD & DSMC \\
\hline $\begin{array}{c}\text { LD-DSMC hybrid } \\
\text { initialized with CFD }\end{array}$ & 161,221 & LD & DSMC \\
\hline
\end{tabular}

In Figure 7, mass density contours are compared. A narrow region of increased density along the shock is observed, arising from post-shock compression. For the DSMC and LD-DSMC solutions, shown in Figure 7-(a), very good agreement is observed. On the other hand, Figure 7-(b) shows density contours of CFD and LD-DSMC. Differences are observed, especially in the wake region.

To see the effects of rarefaction in the wake, profiles of normalized density are shown in Figure 10. Figure 10 presents radial profiles at three axial locations: location a $(Z=0.1524 \mathrm{~m})$ corresponds to the maximum radial dimension of the sphere, location $b(Z=0.2286 \mathrm{~m})$ is on the afterbody, and location $\mathrm{c}(\mathrm{Z}=0.35 \mathrm{~m})$ is in the near wake. The near wake region is shadowed by the forebody $\left(\mathrm{R} / \mathrm{R}_{\mathrm{b}}<1.0\right)$ and the density is generally less than $50 \%$ of the freestream value at locations $b$ and $c$. The results from DSMC and LD-DSMC are almost identical while the CFD results cannot accurately capture the gradient in the bow shock. CFD predicts a wider shock and less peaked density.

Contours of translational and rotational temperature are shown in Figures 8 and 9, respectively. Rotational temperature is presented to demonstrate the presence of rotational-translational non-equilibrium. Very good overall agreement is obtained between the standard DSMC and LD-DSMC results for the translational and rotational temperature contours, as shown in Figure 8-(a) and Figure 9-(a) while the CFD rotational temperatures are noticeably different in the wake (Figure 8-(b), Figure 9-(b)). Within this region, the translational temperature of CFD is underestimated near the wake stagnation line. The disagreement of the rotational temperature between LDDSMC and CFD stands out in the wake region where the continuum assumption breaks down.

Figure 11 presents a comparison of radial profiles for translational temperature at three axial locations of the after body. These are the same locations used in Figure 10. Radial translational temperature profiles show good agreement between DSMC and LD-DSMC, as expected. CFD profiles show the same trends as Figure 10. The bow shock is too wide and a noticeable difference is observed in location c. This discrepancy clearly indicates that the Navier-Stokes formulation becomes inaccurate in the wake, where the flow is very rarefied.

Figure 12-(a) shows contours of the number of particles per cell and Figure 12-(b) shows contours of weight scaling factor. For DSMC, constant weight scaling factor bands are used while adaptive weight scaling factors are used in the LD-DSMC hybrid simulation. Based on the adaptive weight scaling factor, the number of particles per cell is effectively retained around 30 over most of the computational domain.

Since the physical properties are most severe at the stagnation point, it is useful to investigate the profiles along the stagnation streamline. Profiles of the temperature, bulk velocity and mass density along the stagnation streamline are shown in Figure 13. The vertical dotted line denotes the boundaries of the LD and DSMC regions. As expected, the DSMC domain of the hybrid simulation includes high gradient regions within the shock and near the sphere surface. Excellent agreement is obtained between the DSMC and LD-DSMC simulations, while CFD cannot accurately capture the strong gradient in the bow shock. The shock is predicted to be slightly thicker in the DSMC and LD-DSMC solutions in comparison to CFD, and the CFD solution predicts a lower peak translational temperature in the shock. Figures 14 and 15 show profiles of temperature, bulk velocity and mass density along lines inclined at $60^{\circ}$ and $120^{\circ}$ from the freestream direction. Similar to the profiles along the stagnation line, the DSMC 
and LD-DSMC results agree well while the CFD results have significant discrepancies.

Accurate prediction of surface properties is often the most important aspect of these types of computations for hypersonic vehicle design. Figure 16 shows profiles along the sphere surface of heat flux and pressure, respectively. Black and red solid lines represent DSMC and LD-DSMC, respectively, and dotted lines denote CFD results. The maximum gradient length local Knudsen number, $\mathrm{Kn}_{\mathrm{GLL} \max }$, is also shown along the sphere surface. Beyond 90 degrees, $\mathrm{Kn}_{\text {GLL max }}$ increases, which means continuum breakdown occurs. The CFD results show obvious differences where the continuum assumption fails, i.e. in the low-density recirculation region. The DSMC and the LD-DSMC hybrid methods show excellent agreement.

In Table 3, stagnation point heat transfer coefficient and total drag coefficient are compared. The coefficients are calculated according to Equations (5) and (6). The heat flux results contain scatter near the stagnation point. To reduce the statistical noise, the stagnation point heat transfer is averaged over the first ten cells along the surface. The LD-DSMC hybrid method agrees with DSMC to within 5\%. In general, the use of a cutoff value of the continuum breakdown parameter of 0.05 aims to provide agreement between DSMC and the Navier-Stokes equations to within 5\%, so the level of agreement achieved here is considered more than acceptable.

Figure 17 shows percent errors of the LD-DSMC and CFD solutions in comparison to DSMC along the surface. As mentioned above, the DSMC result is considered the most accurate and CFD (Navier-Stokes formulation) becomes inaccurate in the wake where the flow is very rarefied which explains the significant differences (30\%) in those results. The LD-DSMC hybrid simulation agrees with standard DSMC within the target error limit of 5\%.

Table 3. Comparison of surface properties ( $C_{H}$ at the stagnation point, and $C_{D}$ over a surface)

\begin{tabular}{ccccc}
\hline & Standard DSMC & LD-DSMC hybrid & CFD, slip & $\begin{array}{c}\text { LD-DSMC hybrid } \\
\text { initialization with CFD }\end{array}$ \\
\hline $\mathrm{C}_{\mathrm{H}}$ & 0.0974 & $0.0976(0.205 \%)$ & $0.0951(-2.36 \%)$ & $0.0955(-1.95 \%)$ \\
\hline $\mathrm{C}_{\mathrm{D}}$ & 0.968 & $0.967(-0.103 \%)$ & $0.965(-0.310 \%)$ & $0.954(-1.45 \%)$ \\
\hline \multicolumn{5}{c}{$\mathrm{C}_{\mathrm{H}}=\frac{2 \mathrm{q}}{\mathrm{\rho} V_{\infty}^{\mathrm{s}}}$} \\
$\mathrm{C}_{\mathrm{D}}=\frac{2 \mathrm{D}}{\rho V_{\infty}^{2} \mathrm{~S}}$
\end{tabular}

In Reference 19, the computational performance of the LD-DSMC hybrid method is discussed. The hybrid method does not show any computational expense reduction over simulations performed using standard DSMC on the same mesh. Therefore, the hybrid simulation is performed here on a coarser mesh that provides grid independence in the LD calculations, but is considerably less refined than necessary to meet DSMC guidelines to reduce the computational expense. As discussed earlier, during collision selection routines as part of DSMC calculations, each cell in the DSMC domain is divided into a number of subcells.

A coarser mesh (total number of cells: 234,256) is employed for the hybrid LD-DSMC calculation while a denser mesh (total number of cells: 1,071,858) is employed for the standard DSMC calculation. Generally, the total number of particles is one of the main factors affecting the computational performance. In the LD-DSMC hybrid calculation, with an adaptive weight scaling factor, each cell is controlled to have between 20 and 30 particles by updating the weight scaling factor. The total number of particles is $16 \mathrm{M}$ and $73 \mathrm{M}$ for LD-DSMC and DSMC, respectively. The DSMC and LD-DSMC simulations are considered to have reached steady-state when the total number of simulation particles no longer varies appreciably. In this way, steady state is found to occur after 150,000 time steps and the simulation is then continued to build up a large sample size for an additional 150,000 time steps. All simulations are performed in parallel on a cluster at the University of Michigan. The LD-DSMC hybrid method shows computational expense reduction over simulations performed using standard DSMC. Total simulation times are approximately 1880 hours for the standard DSMC (Table 4). The computational cost of the LD-DSMC simulation is about 1520 hours; this is $20 \%$ less the standard DSMC calculation. CFD requires about one quarter of the cost of LD-DSMC.

\section{B. LD-DSMC Initialized with a Navier-Stokes solution}

LD-DSMC initialized with a NS solution is evaluated by comparing with the conventional LD-DSMC hybrid method. The simulation is performed for the same flow condition described in Table 1. The LD-DSMC hybrid simulation initialized with CFD begins with the method decomposition shown in Figure 18-(left) that plots contours 
of maximum gradient length local Knudsen number, $\mathrm{Kn}_{\mathrm{GLL} \max }$. The red contour indicates the region where $\mathrm{Kn}_{\text {GLL max }}$ is larger than 0.05 , that is simulated with DSMC, and the other regions are simulated with LD. To be used for particle simulation, the initial CFD mesh must be refined.

In the simulation, the mesh refinement is performed based on the value of $\mathrm{Kn}_{\text {GLL max }}$ of 0.0001 . The cells where $\mathrm{Kn}_{\text {GLL max }}$ is larger than 0.0001 are refined based on the cell-centered value of mean free path. A maximum refinement factor is restricted to 5 to avoid excessive refinement which may decrease computational efficiency. Figure 18-(right) shows the number of refinements of each CFD cell. More refined cells exist along the shock and the wake region. Figure 19 shows the final mesh for the LD-DSMC hybrid simulation. Smaller cells are distributed along the shock and the wake region. The total numbers of cells for various meshes are listed in Table 2. The total number of cells for LD-DSMC initialized with CFD is smaller than LD-DSMC because mean free path based refinement helps to avoid large numbers of small cells.

Figure 20 shows continuum breakdown domain boundaries of the LD-DSMC hybrid simulation initialized with CFD. As expected, the LD domain includes high-density near-equilibrium regions in the freestream, aftershock region, and the wake. The bow shock dissipates eventually under the rarefied flow condition, and the whole bow shock is not captured in the DSMC region especially far away from the sphere.

The LD-DSMC hybrid initialized with NS flowfield solution agrees well with the other two simulations. Figure 16 shows surface properties. Detailed quantative analysis is provided in Table 3 and Figure 17. Over the entire sphere surface, the error is within the limit of 5\%. Table 3 lists stagnation point heat transfer coefficient and total drag coefficient over the entire sphere. Again, LD-DSMC initialization with NS solution agrees very well within the error limit of $5 \%$.

Table 4. Computational efficiency study of DSMC, LD-DSMC, CFD, and LD-DSMC initialized with CFD

\begin{tabular}{ccccc}
\hline & $\begin{array}{c}\text { Standard } \\
\text { DSMC }\end{array}$ & LD-DSMC hybrid & CFD, slip & $\begin{array}{c}\text { LD-DSMC initialized } \\
\text { with CFD }\end{array}$ \\
\hline Total number of cells & $1,071,858$ & 234,256 & 100,000 & 161,221 \\
$\begin{array}{c}\text { Number of DSMC cells } \\
\text { (LD cells) }\end{array}$ & & 100,525 & & 101,819 \\
$\begin{array}{c}\text { Total simulation } \\
\text { particles }\end{array}$ & $73 \mathrm{M}$ & $(133,731)$ & & $(59,402)$ \\
$\begin{array}{c}\text { Computational } \\
\text { cost/particle/iteration } \\
\text { Total CPU hours }\end{array}$ & $2.61 \times 10^{-7}$ & $7.02 \times 10^{-7}$ & & $7.5 \mathrm{M}$ \\
Relative cost & 1880 hours & 1520 hours & 320 hours & $7.30 \times 10^{-7}$ \\
\hline
\end{tabular}

In Table 4, computational efficiencies of the four cases are compared. The total number of cells for LD-DSMC initialized with NS solution is 161,211 and the total number of particles is $9.5 \mathrm{M}$. The average numbers of particles per cell are 68 and 56 for LD-DSMC and LD-DSMC initialized with NS, respectively. For a fair comparison, an identical average number of particles per cell should be used. However, average number of particles per cell depends on the size of cells and number of subcells. In every simulation, the desired number of particles per cell is set to 30 . The reason why the actual average number of particles per cell is larger than 30 is due to the subcell utility. Each subcell has a desired number of particles per subcell (set to 4). If some cells are much larger than the mean free path, they may have a large number of subcells and a larger number of particles than 30. The computational cost per particle per iteration of LD is $1.19 \times 10^{-6} \mathrm{sec}$ and $2.61 \times 10^{-7} \mathrm{sec}$ for DSMC. The LD method has the largest computation cost per particle per iteration among the methods. When the LD-DSMC simulation is initialized with a NS solution, the LD-DSMC hybrid simulation requires $56 \%$ of the cost of the conventional LD-DSMC simulation, or $45 \%$ of the cost of standard DSMC.

\section{Conclusions}

The LD-DSMC hybrid method is an all-particle, multiscale approach that allows for very strong coupling between continuum and non-continuum flow regimes. The main goal of this study was to evaluate the accuracy and efficiency of the LD-DSMC hybrid simulation. LD-DSMC can reproduce full-DSMC results at a fraction of the cost. The LD-DSMC hybrid method was applied to an axisymmetric hypersonic blunt body flow problem where the global Knudsen number was 0.002 . The method successfully described flow characteristics within 5\% error compared with standard DSMC as a reference. Also, the LD-DSMC hybrid solution showed much higher accuracy than the CFD solution. In addition, the LD-DSMC hybrid method decreased computational cost by up to $20 \%$. 
A module initializing the LD-DSMC hybrid method with a Navier-Stokes solution was developed. It was used in place of the conventional LD-DSMC hybrid algorithm to increase computational efficiency. Based on the initial CFD solution, $\mathrm{Kn}_{\mathrm{GLL}} \max$ is used to determine domain regimes, and then mesh refinement is performed to give proper cell size for DSMC and LD calculations in each computational domain. The LD-DSMC hybrid method reduced the computational cost by $45 \%$ by using the initializing module based on a Navier-Stokes solution. In conclusion, application to a hypersonic sphere flow clearly shows that the LD-DSMC hybrid approach is very promising for the solution of steady state flows where a wide range of local Knudsen number is evaluated.

\section{Acknowledgment}

Financial support for this work was provided by NASA through grant NNX08AD02A.

${ }^{1}$ Http://Www.Nasa.Gov.

\section{References}

${ }^{2}$ Hubbard, G. S., "The Exploration of Mars; Historical Context \& Current Results," AIAA Paper 2004-3, 42nd AIAA Aerospace Sciences Meeting and Exhibit, Reno, NV, Jan. 2004.

${ }^{3} \mathrm{Http}: / /$ Solarsystem.Nasa.Gov/Index.Cfm.

${ }^{4}$ Anderson Jr, J. D., Modern compressible flow with historical perspective, McGraw-Hill, New York, 1990.

${ }^{5}$ Moss, J. N., Blanchard, R. C., Wilmouth, R. G. and Braun, R. D., "MARS Pathfinder Rarefied Aerodynamics: Computations and Measurements," AIAA Paper 1998-298, 36th AIAA Aerospace Sciences Meeting and Exhibit, Reno, NV, Jan. 1998.

${ }^{6}$ Moss, J. N., Wilmoth, R. G. and Price, J. M., "DSMC Simulation of Blunt Body Flows for Mars Entries: Mars Pathfinder and Mars Microprobe Capsules," AIAA Paper 1997-2508, 32nd Thermophysics Conference, Atlanta, GA, June 1997.

${ }^{7}$ Gnoffo, P. A., "Planetary-Entry Gas Dynamics 1," Annual Review of Fluid Mechanics, Vol. 31, No. 1, 1999, pp. 459-494.

${ }^{8}$ Bird, G. A., Molecular Gas Dynamics and the Direct Simulation of Gas Flows, Oxford University Press, New York, 1994.

${ }^{9}$ Tiwari, S. and Klar, A., "An adaptive Domain Decomposition Procedure for Boltzmann and Euler Equations," Journal of Computational Applied Mathematics, Vol. 90, No.2, 1998, pp. 223-237.

${ }^{10}$ Macrossan, M. N., "A Particle-Only Hybrid Method for Near-Continuum Flows," 22nd International Symposium on Rarefied Gas Dynamics, Vol. 585, 2001, pp. 388-395.

${ }^{11}$ Bartel, T. J., Sterk, T. M., Payne, J. and Preppernau, B., "DSMC Simulation of Nozzle Expansion Flow Fields," AIAA Paper 1994-2047, 6th Joint Thermophysics and Heat Transfer Conference, Colorado Springs, CO, June 1994.

${ }^{12}$ Kolobov, V. I., Arslanbekov, R. R., Aristov, V. V., Frolova, A. A. and Zabelov, S. A., "Unified Solver for Rarefied and Continuum Flows with Adaptive Mesh and Algorithm Refinement," Journal of Computational Physics, Vol. 223, No. 2, 2007, pp. 589-608.

${ }^{13}$ Titov, E. V., Zeifman, M. I. and Levin, D. A., "Application of the Kinetic and Continuum Techniques to the Multi-Scale Flows in MEMS Devices," AIAA Paper 2005-1399, 43rd AIAA Aerospace Sciences Meeting and Exhibit, Reno, NV, Jan. 2005.

${ }^{14}$ Breuer, K. S., Piekos, E. S. and Gonzales, D. A., "DSMC Simulations of Continuum Flows," AIAA Paper 1995-2088, 30th AIAA Thermophysics Conference, San Diego, CA, June 1995.

${ }^{15}$ Pullin, D. I., "Direct Simulation Methods for Compressible Inviscid Ideal-Gas Flow," Journal of Computational Physics, Vol. 34, No. 2, 1980, pp. 231-244.

${ }^{16}$ Burt, J. M. and Boyd, I. D., "Extension of a Multiscale Particle Scheme to Near-Equilibrium Viscous Flows," AIAA Paper 2008-3914, 40th Thermophysics Conference, Seattle, WA, June 2008.

${ }^{17}$ Burt, J. M. and Boyd, I. D., "A Low Diffusion Particle Method for Simulating Compressible Inviscid Flows," Journal of Computational Physics, Vol. 227, 2008, pp. 4653-4670.

${ }^{18}$ Jun, E., Burt, J. M. and Boyd, I. D., "All-Particle Multiscale Computation of Hypersonic Rarefied Flow," 27th International Symposium on Rarefied Gas Dynamics, Vol. 1333, 2011, pp. 557.

${ }^{19}$ Jun, E., Burt, J. M. and Boyd, I. D., "All-Particle Multiscale Computation of Hypersonic Rarefied Flow," AIAA Paper 2010-0822, 48th AIAA Aerospace Sciences Meeting and Exhibit, Orlando, FL, Jan. 2010.

${ }^{20}$ Burt, J. M. and Boyd, I. D., "A Hybrid Particle Approach for Continuum and Rarefied Flow Simulation," Journal of Computational Physics, Vol. 228, No. 2, 2009, pp. 460-475.

${ }^{21}$ Burt, J. M. and Boyd, I. D., "A Hybrid Particle Scheme for Simulating Multiscale Gas Flows with Internal 
Energy Nonequilibrium," AIAA Paper 2010-0820, 48th AIAA Aerospace Sciences Meeting and Exhibit, Orlando, FL, Jan. 2010.

${ }^{22}$ Boyd, I. D. and Chen, G., "Predicting Failure of the Continuum Fluid Equations in Transitional Hypersonic Flows," Physics of Fluids, Vol. 7, No.1, 1994, pp. 210-219.

${ }^{23}$ Wang, W. L. and Boyd, I. D., "Predicting Continuum Breakdown in Hypersonic Viscous Flows," Physics of Fluids, Vol. 15, No. 1, 2003, pp. 91-100.

${ }^{24}$ Burt, J. M. and Boyd, I. D., "Rotational and Vibrational Nonequilibrium in a Low Diffusion Particle Method for Continuum Flow Simulation," AIAA Paper 2009-3743, 41st AIAA Thermophysics Conference, San Antonio, TX, June 2009.

${ }^{25}$ Borgnakke, C. and Larsen, P. S., "Statistical Collision Model for Monte Carlo Simulation of Polyatomic Gas Mixture," Journal of Computational Physics, Vol. 18, No.3, 1975, pp. 405-420.

${ }^{26}$ Millikan, R. C. and White, D. R., "Systematics of Vibrational Relaxation," Journal of Chemical Physics, Vol. 39, 1963, pp. 3209-3213.

${ }^{27}$ Boyd, I. D. and Wang, W. L., "Monte Carlo Computations of Hypersonic Interacting Flows," AIAA Paper 2001-1029, 39th AIAA Aerospace Science Meeting and Exhibit, Reno, NV, Jan. 2001.

${ }^{28}$ Martin, A., Scalabrin, L. C. and Boyd, I. D., "High Performance Modeling of Atmospheric Re-entry Vehicles," Journal of Physics: Conference Series, Vol. 341, 2012 , Article 012002.

${ }^{29}$ Lofthouse, A. J., Scalabrin, L. C. and Boyd, I. D., "Velocity Slip and Temperature Jump in Hypersonic Aerothermodynamics," Journal of Thermophysics and Heat Transfer, Vol. 22, No. 1, 2008, pp. 38-49.

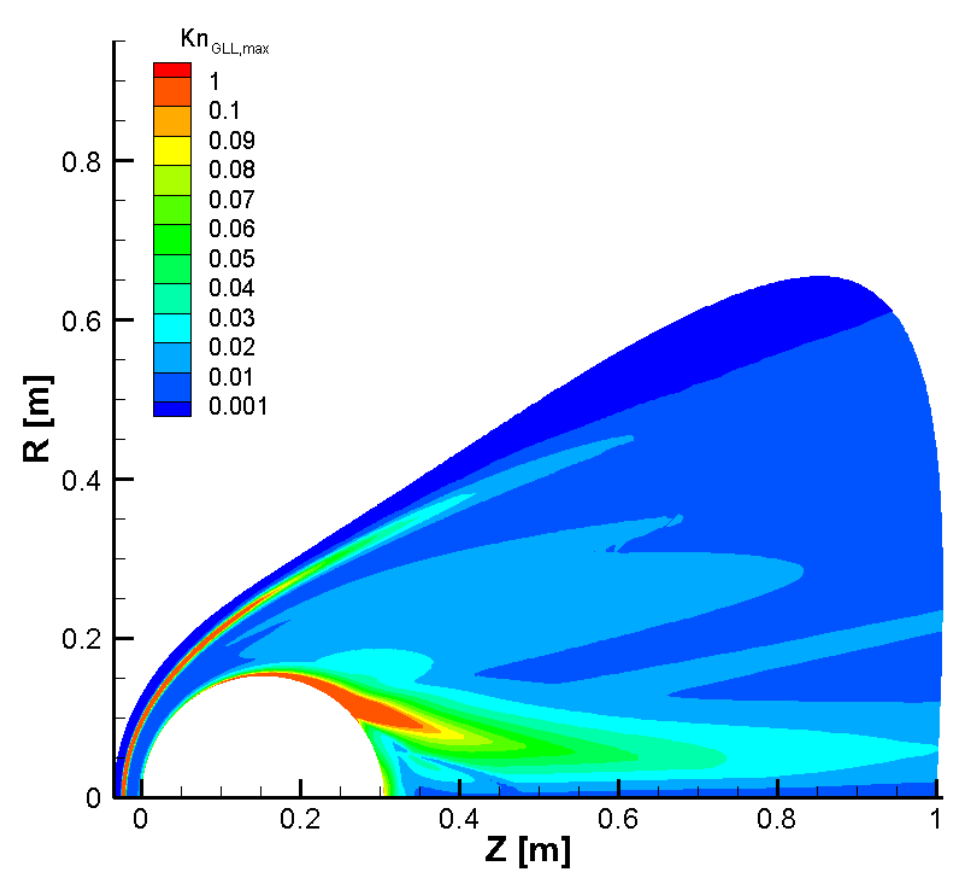

Figure 1. An example of multiscale flow mixed with continuum and non-continuum regions: Maximum gradient length local Knudsen number $\left(\mathrm{Kn}_{\mathrm{GLL} \max }\right)$ contours 

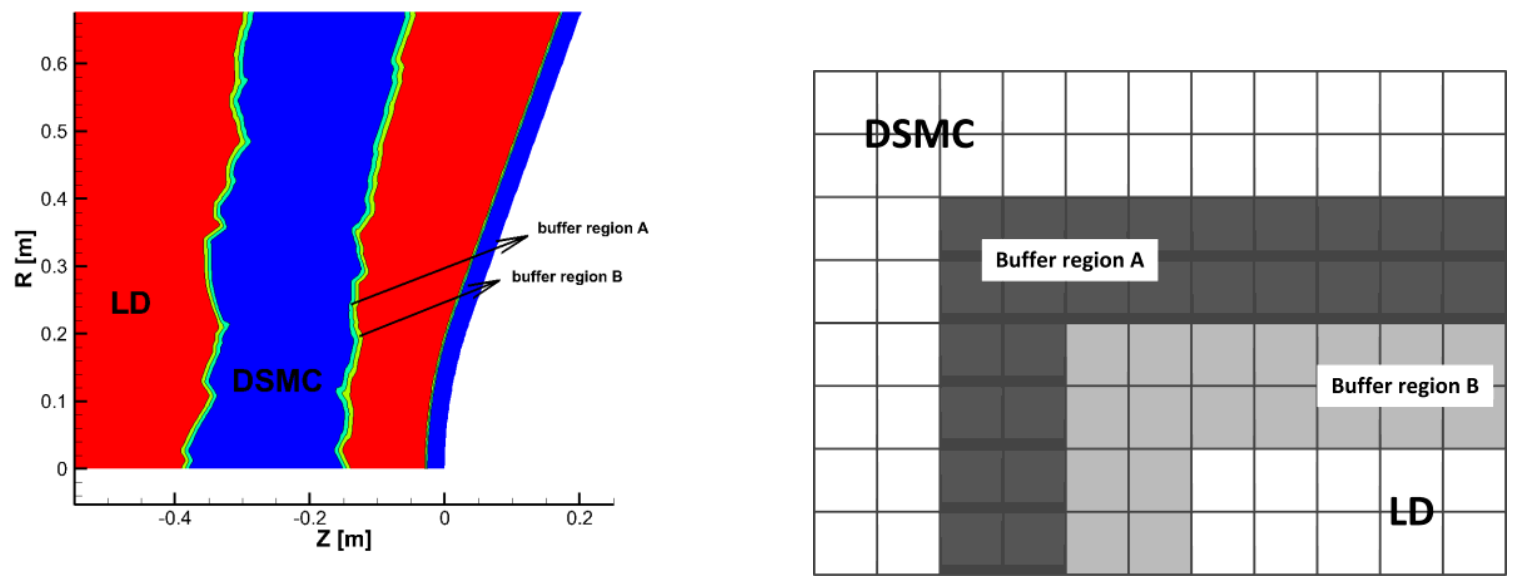

Figure 2. Location of buffer regions in a hybrid simulation

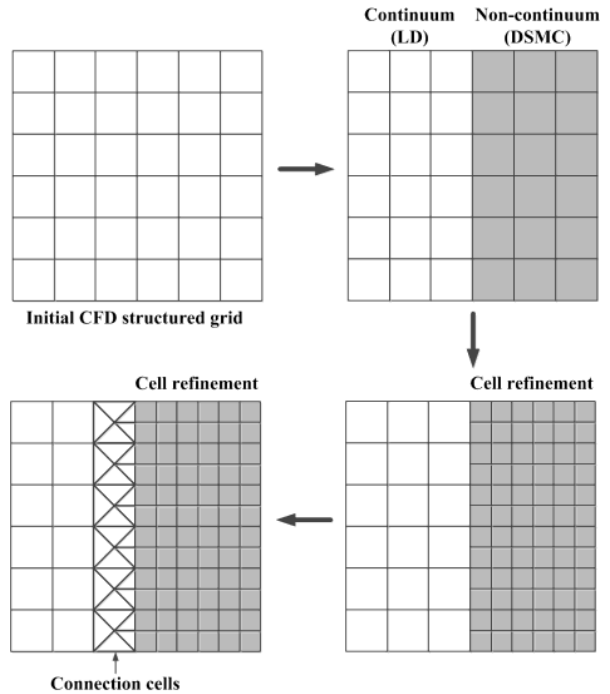

(a) Procedure of cell refinement

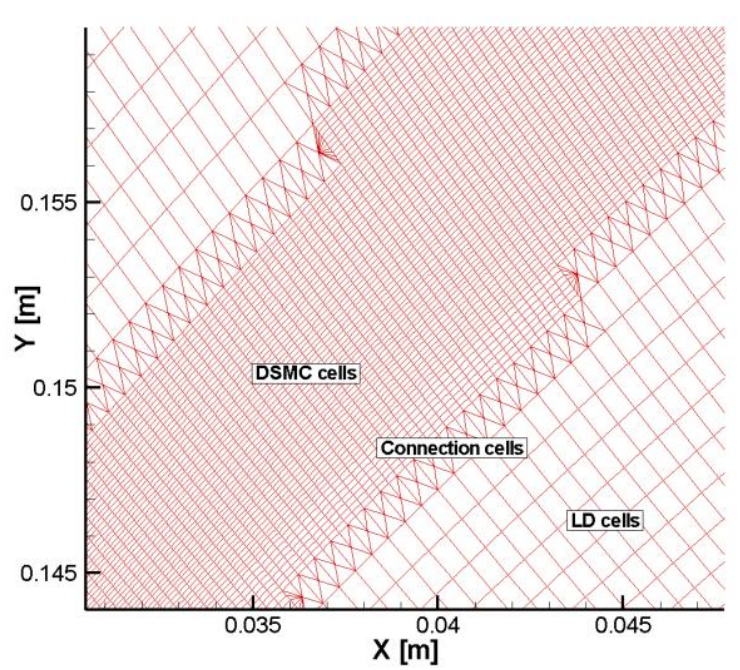

(b) Refined mesh for the LD-DSMC hybrid simulation

Figure 3. Cell refinement 

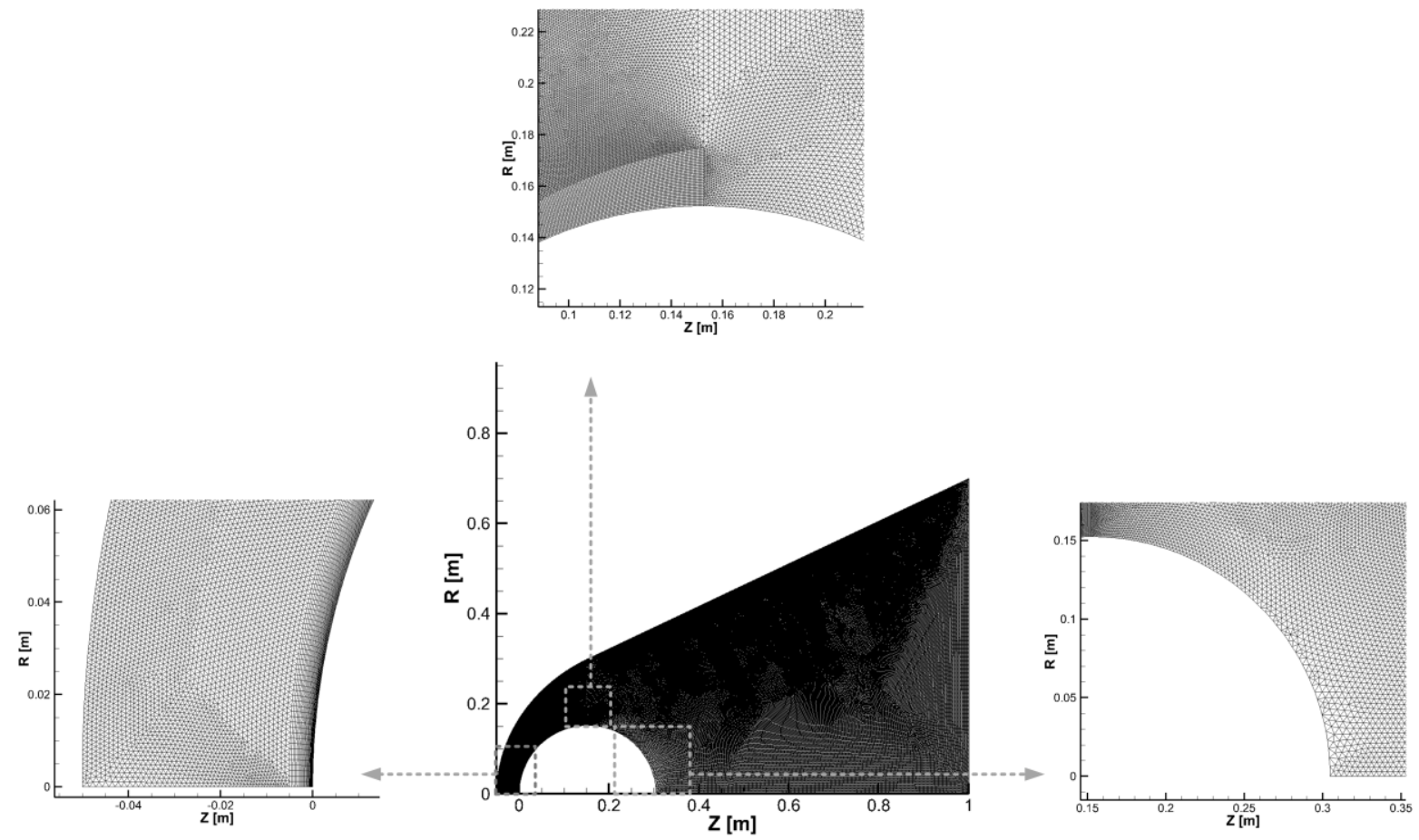

Figure 4. Grid configuration for hypersonic flow over a sphere

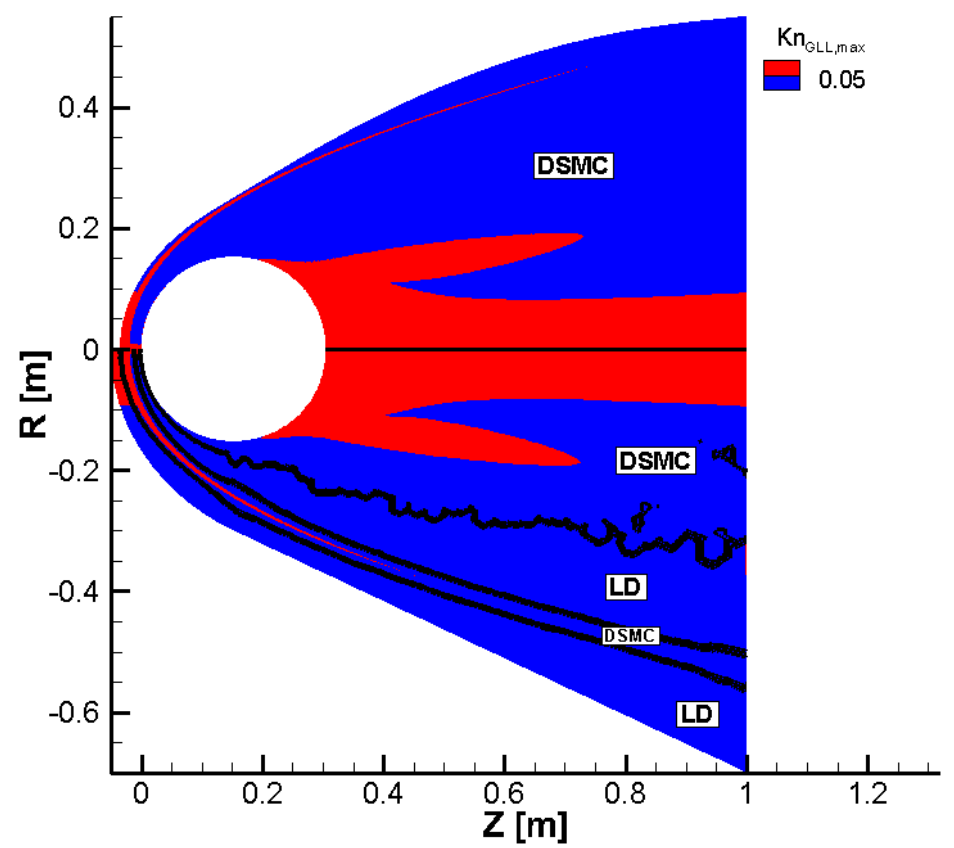

Figure 5. Contours of maximum gradient length local Knudsen number, $\mathrm{Kn}_{\mathrm{GLL} \max }$, and domain decomposition region (upper: DSMC, lower LD-DSMC hybrid)

13

American Institute of Aeronautics and Astronautics 

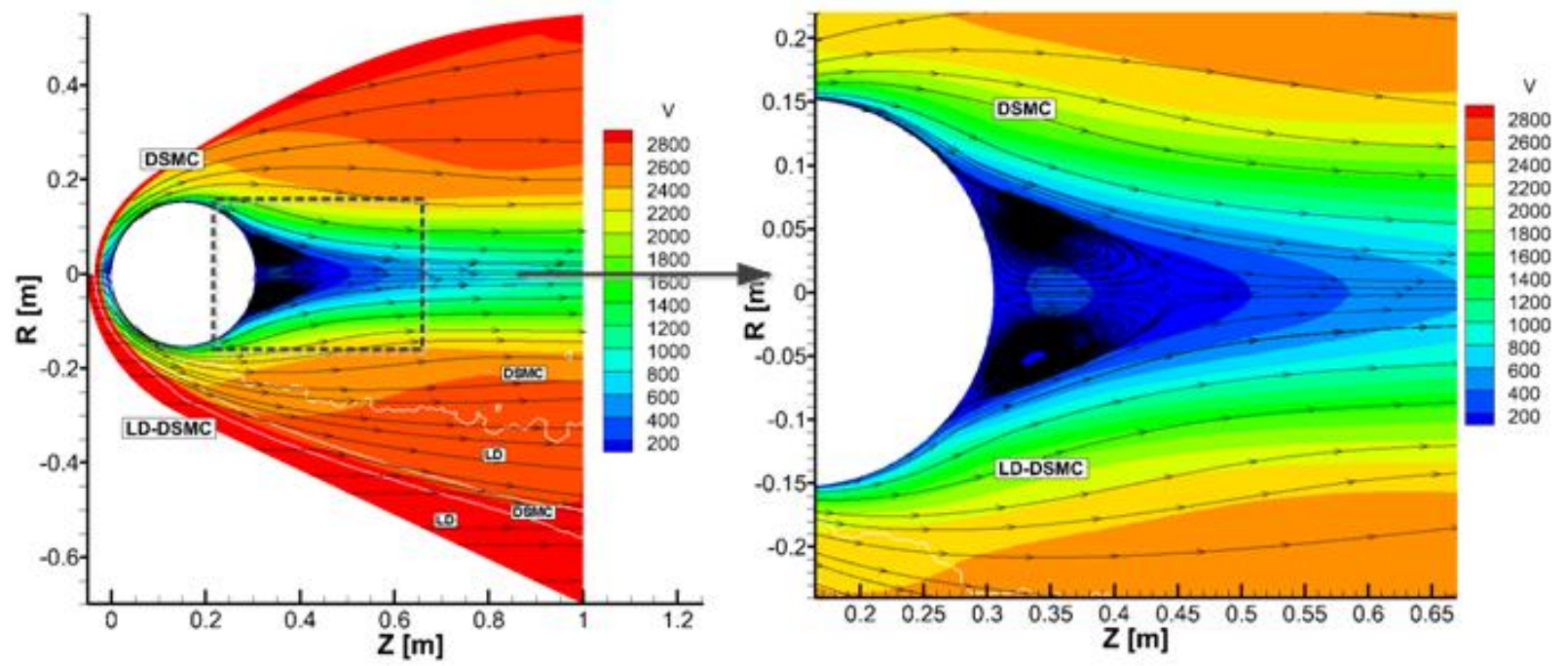

(a) DSMC (upper) and LD-DSMC (lower)
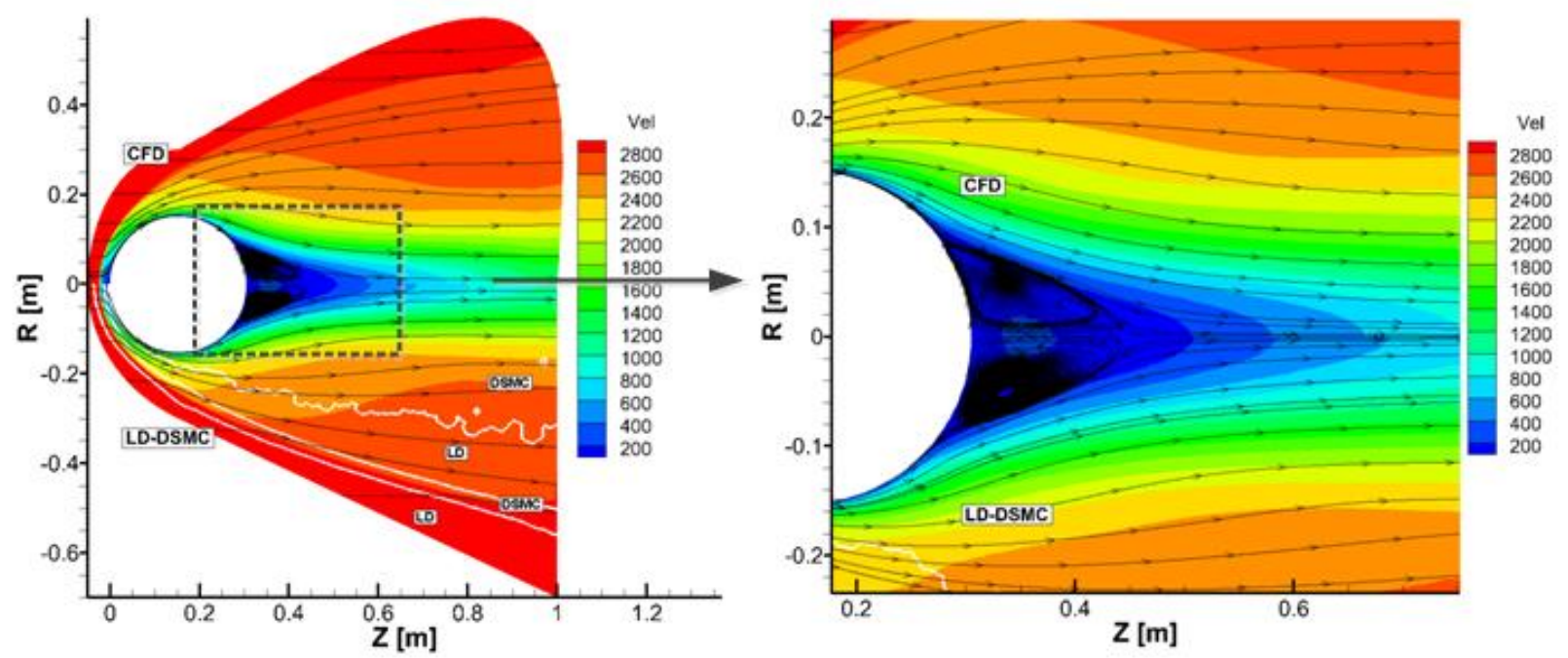

(b) CFD (upper) and LD-DSMC (lower)

Figure 6. Recirculation region and velocity contours $[\mathrm{m} / \mathrm{s}]$ 


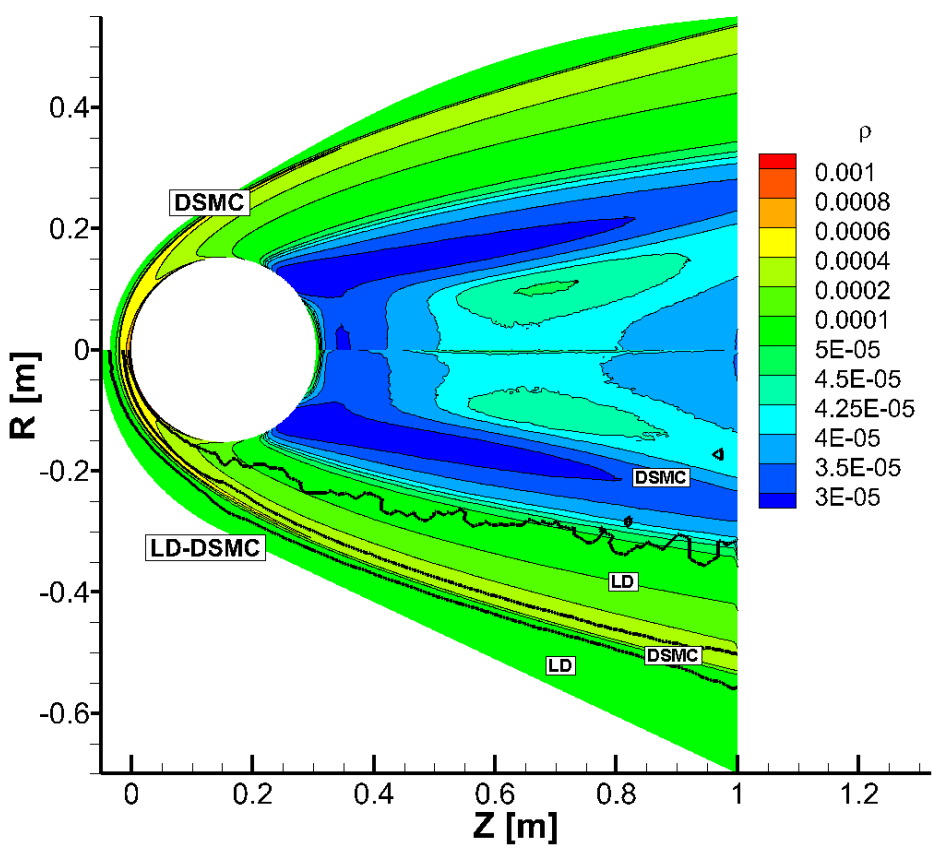

(a) upper: DSMC, lower: LD-DSMC

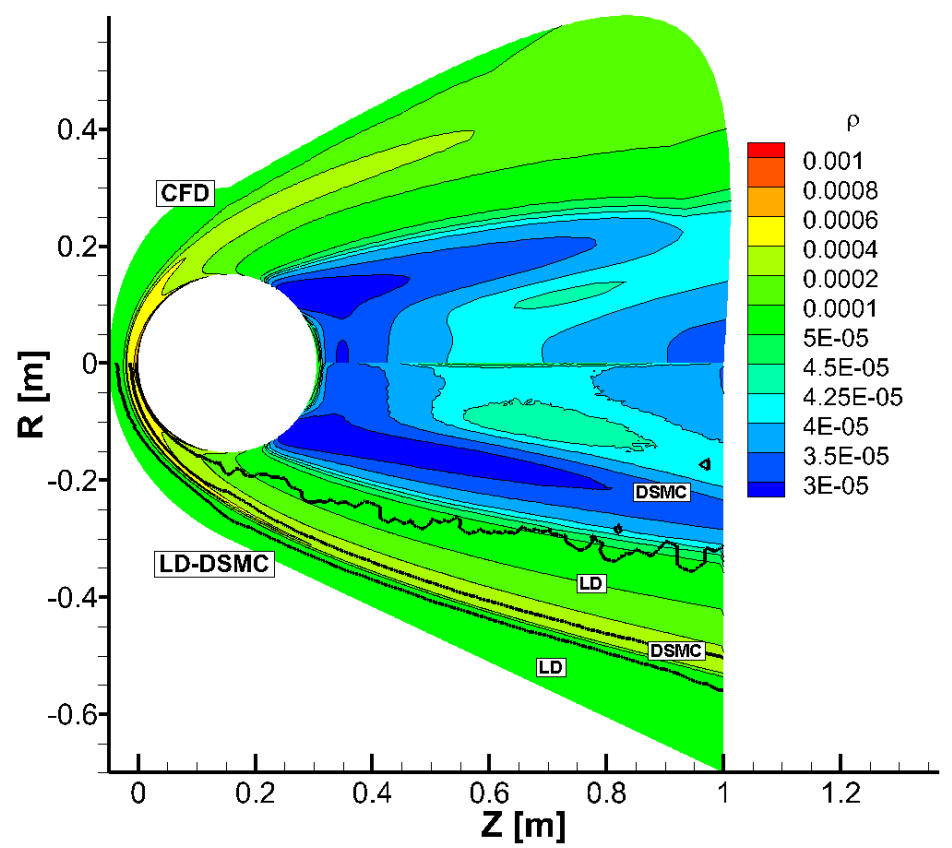

(b) upper: CFD lower: LD-DSMC

Figure 7. Density contours of $\mathrm{N}_{2}$ hypersonic flow over a sphere $\left[\mathrm{kg} / \mathrm{m}^{\mathrm{a}}\right]$ 


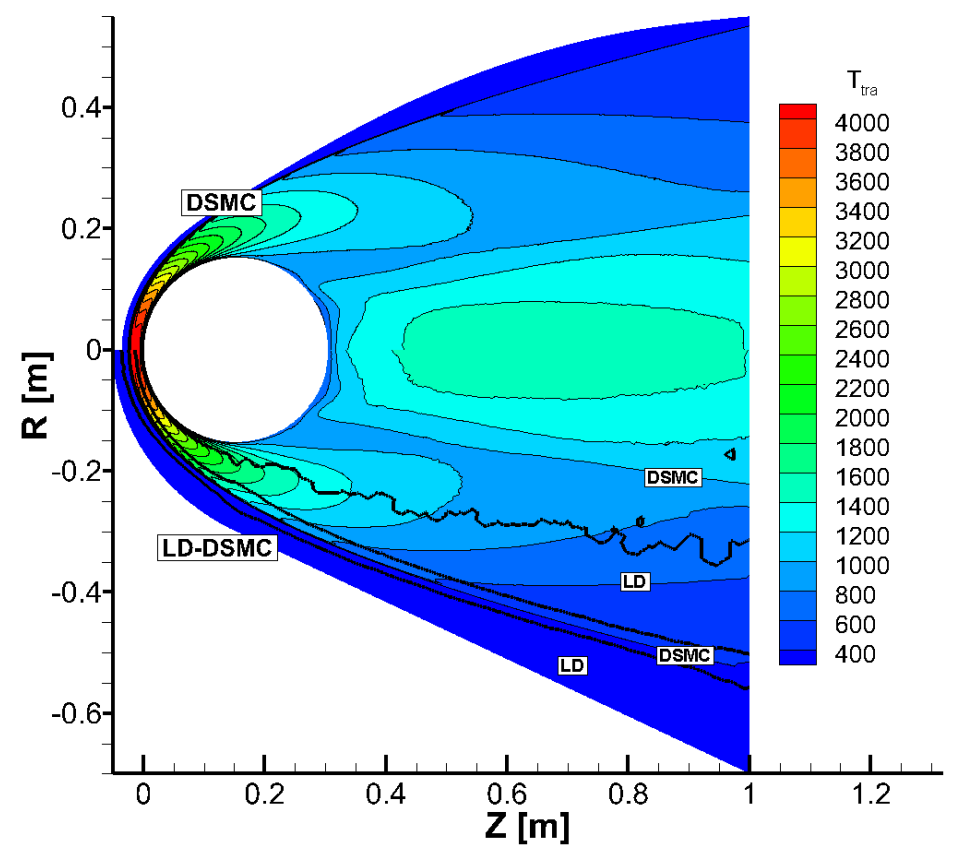

(a) upper: DSMC, lower: LD-DSMC

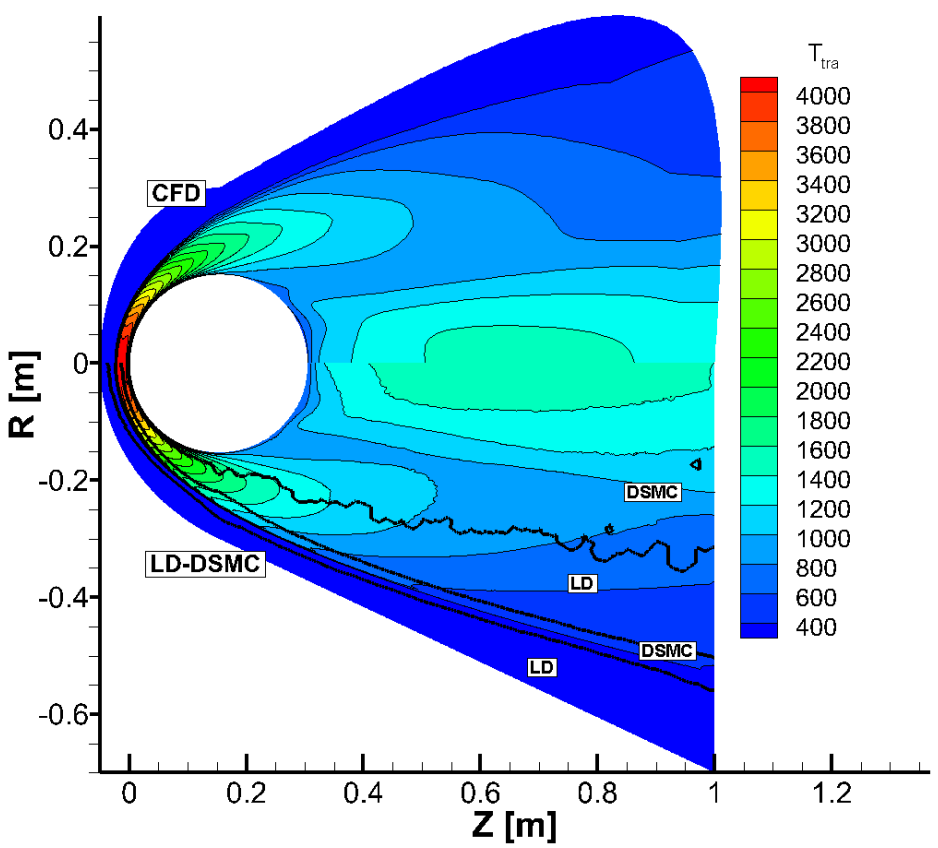

(b) upper: CFD lower: LD-DSMC

Figure 8. Translational temperature contours of $\mathrm{N}_{2}$ hypersonic flow over a sphere [K] 


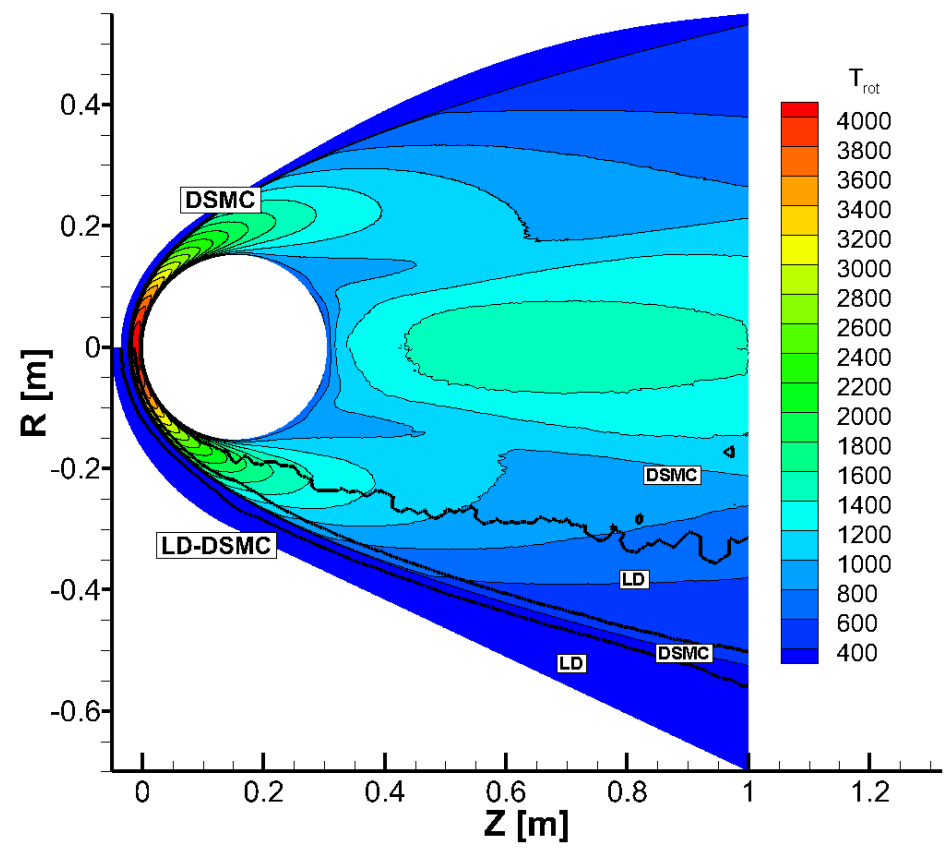

(a) upper: DSMC, lower: LD-DSMC

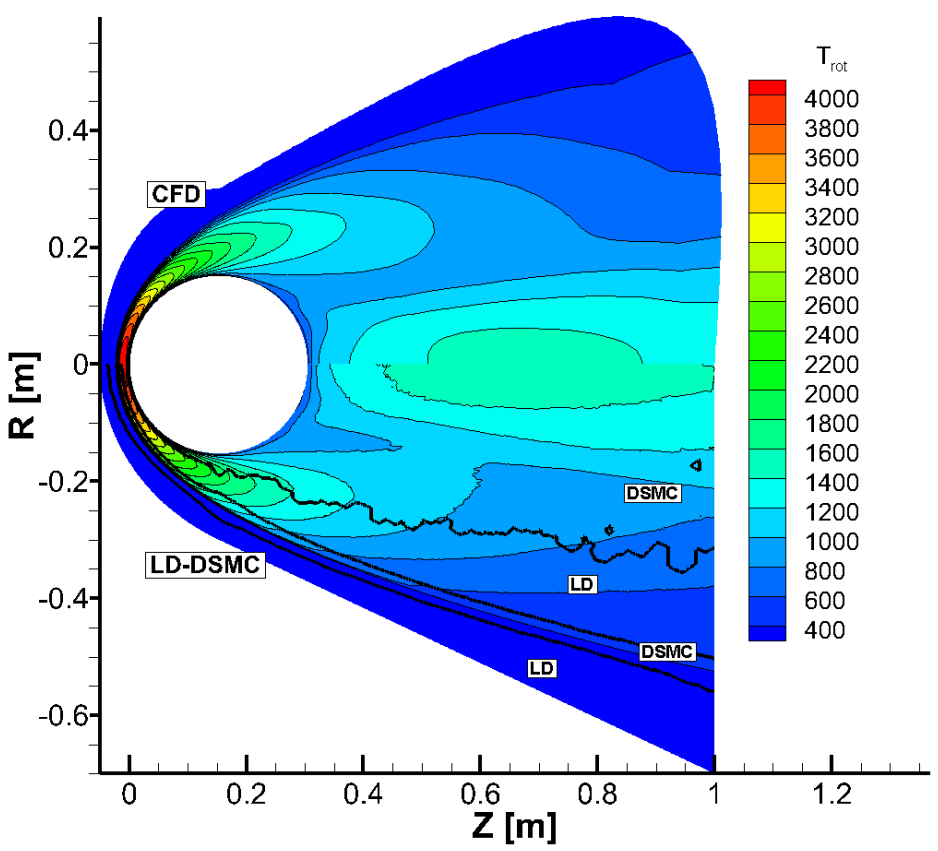

(b) upper: CFD lower: LD-DSMC

Figure 9. Rotational temperature contours of $\mathbf{N}_{2}$ hypersonic flow over a sphere [K] 


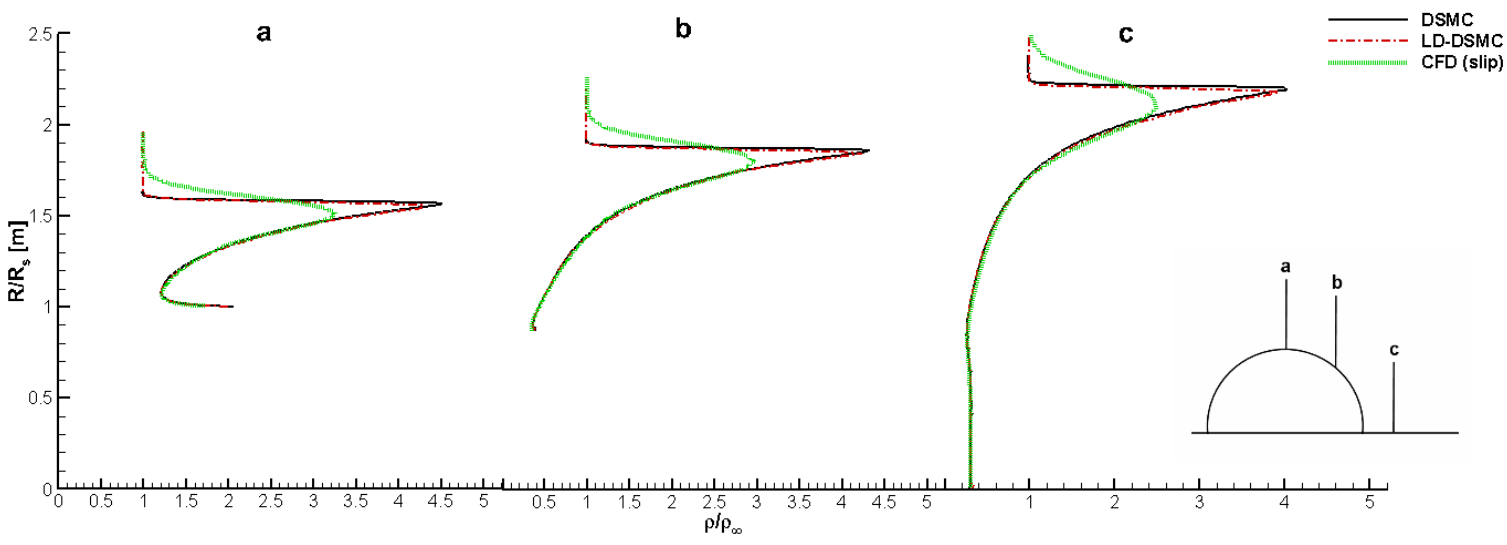

Figure 10. Non-dimensional density profiles of DSMC, LD-DSMC and CFD results near the wake region

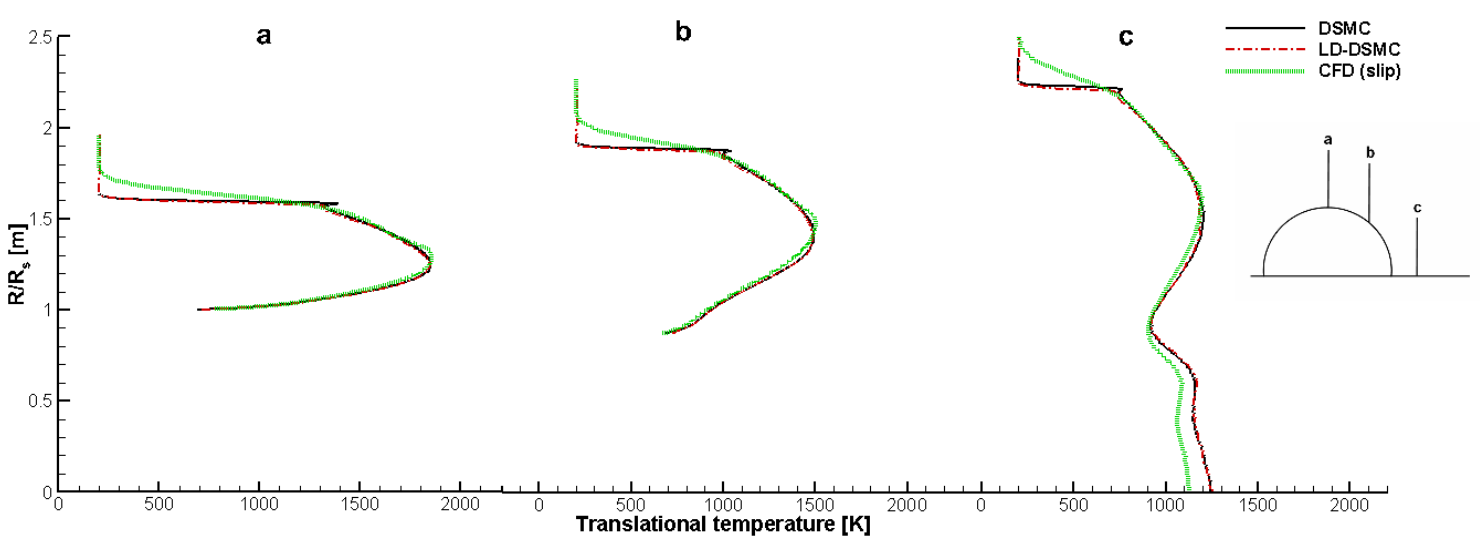

Figure 11. Translational temperature profiles of DSMC, LD-DSMC and CFD results near the wake region 


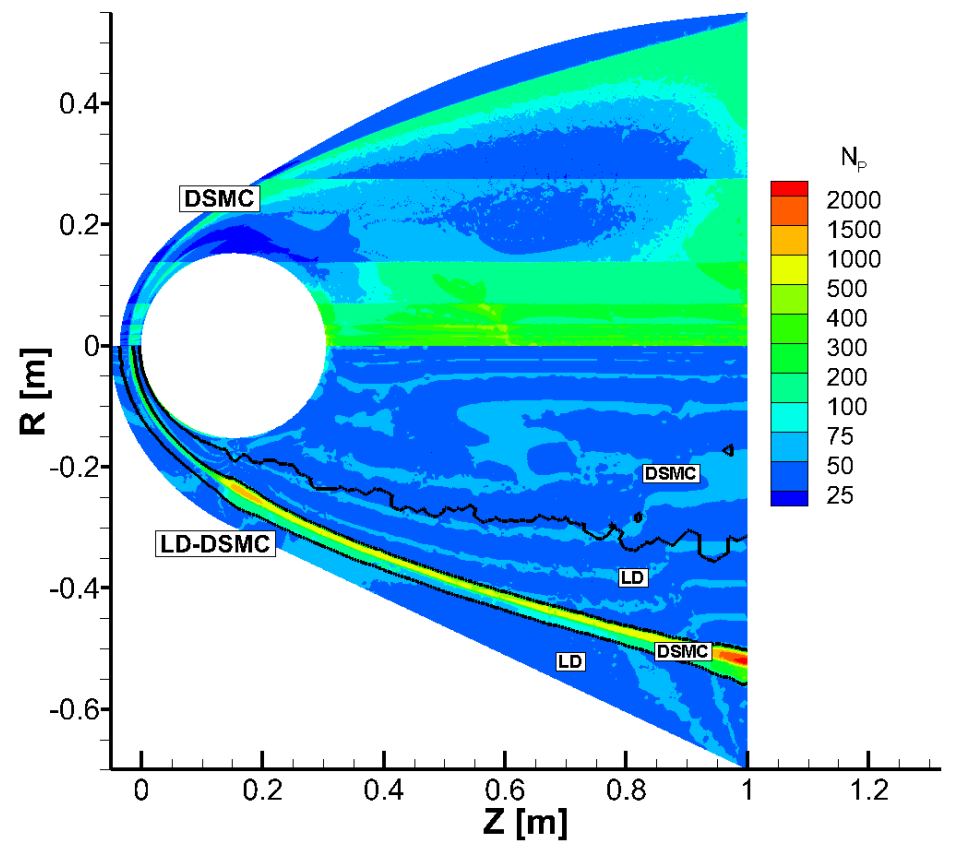

(a) Number of particles per cell (upper: DSMC, lower: LD-DSMC)

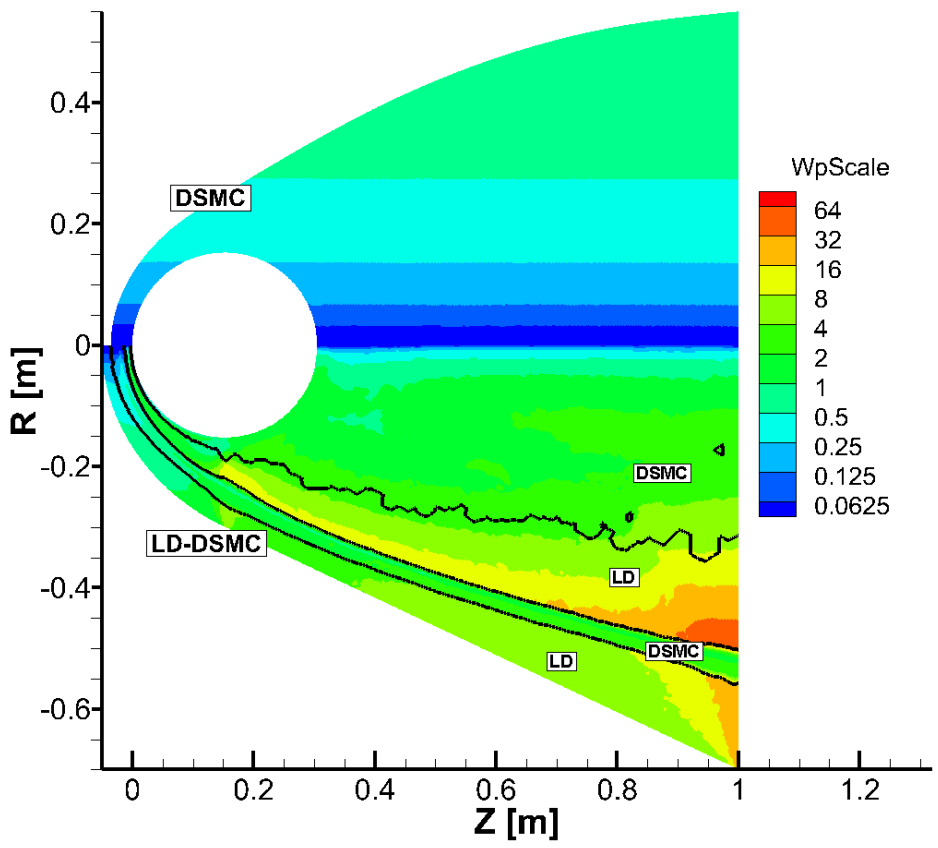

(b) Weight scaling factor (upper: DSMC, lower: LD-DSMC)

Figure 12. Number of particles per cell and weight scaling factor 


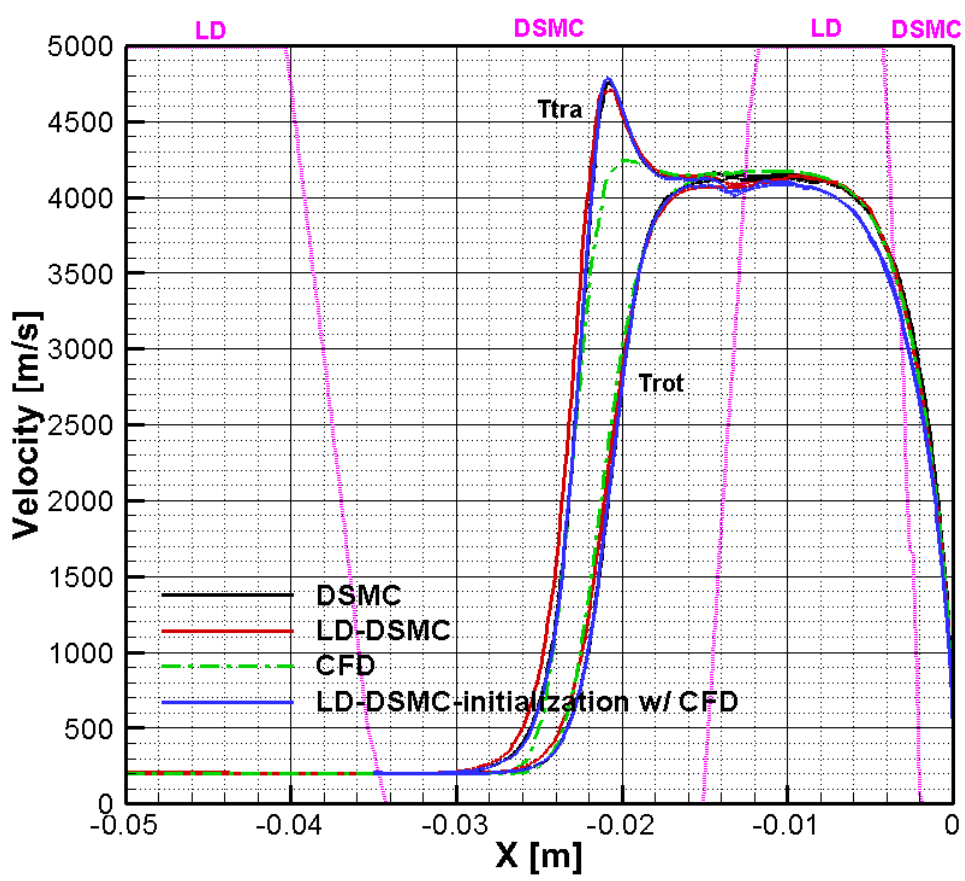

(a) Profiles of translational and rotational temperatures

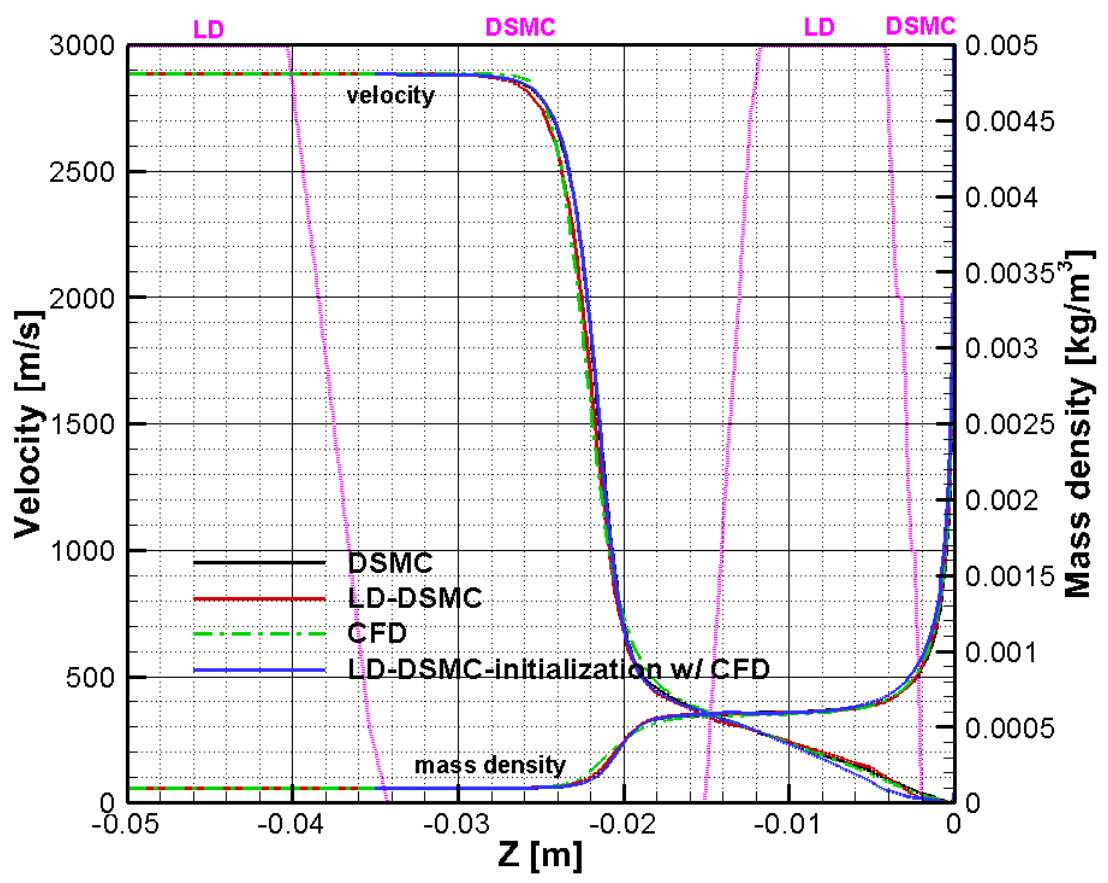

(b) Profiles of bulk velocity and mass density

Figure 13. Profiles along the stagnation line: Black and green lines represent standard DSMC and the conventional LD-DSMC hybrid, respectively. Dotted green lines denote CFD results. Blue lines are the LD-DSMC hybrid simulation initialized with Navier-Stokes solution.

American Institute of Aeronautics and Astronautics 


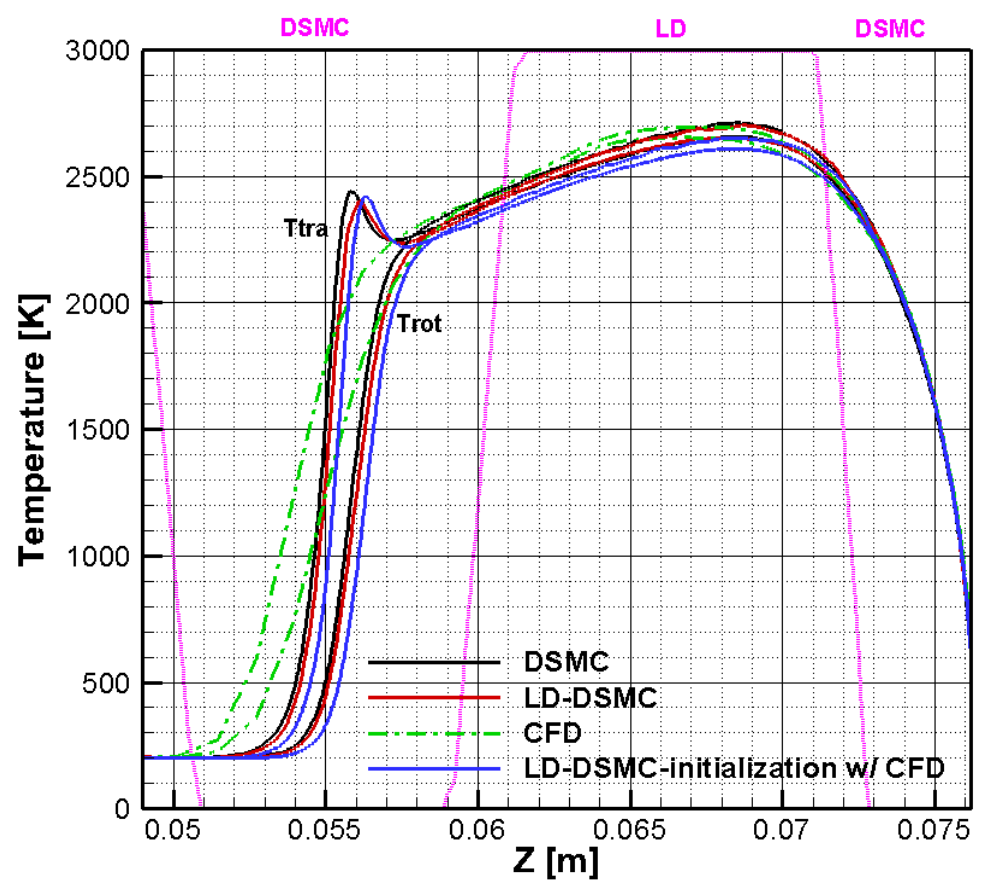

(a) Profiles of temperatures

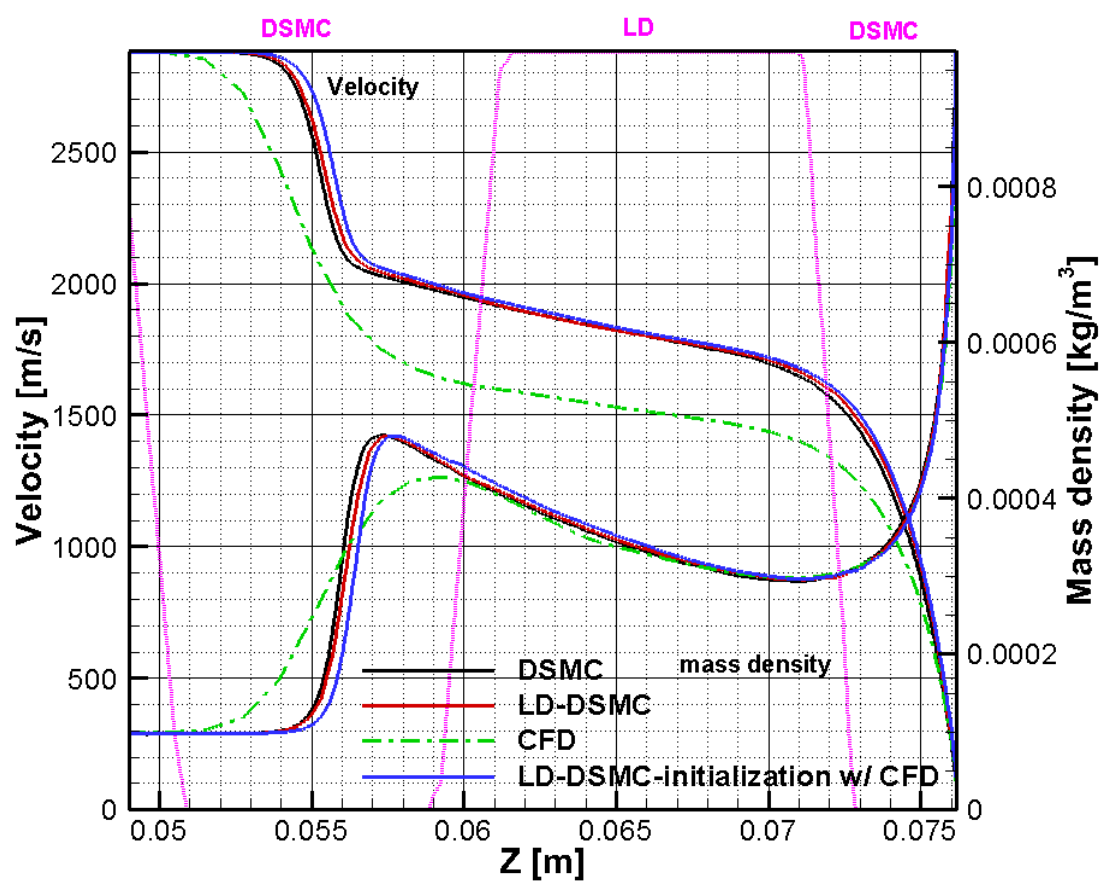

(b) Profiles of bulk velocity and mass density

Figure 14. Profiles along an extraction line inclined at $60^{\circ}$ from the freestream direction: Black and green lines represent standard DSMC and the conventional LD-DSMC hybrid, respectively. Dotted green lines denote CFD results. Blue lines are the LD-DSMC hybrid simulation initialized with Navier-Stokes solution. 


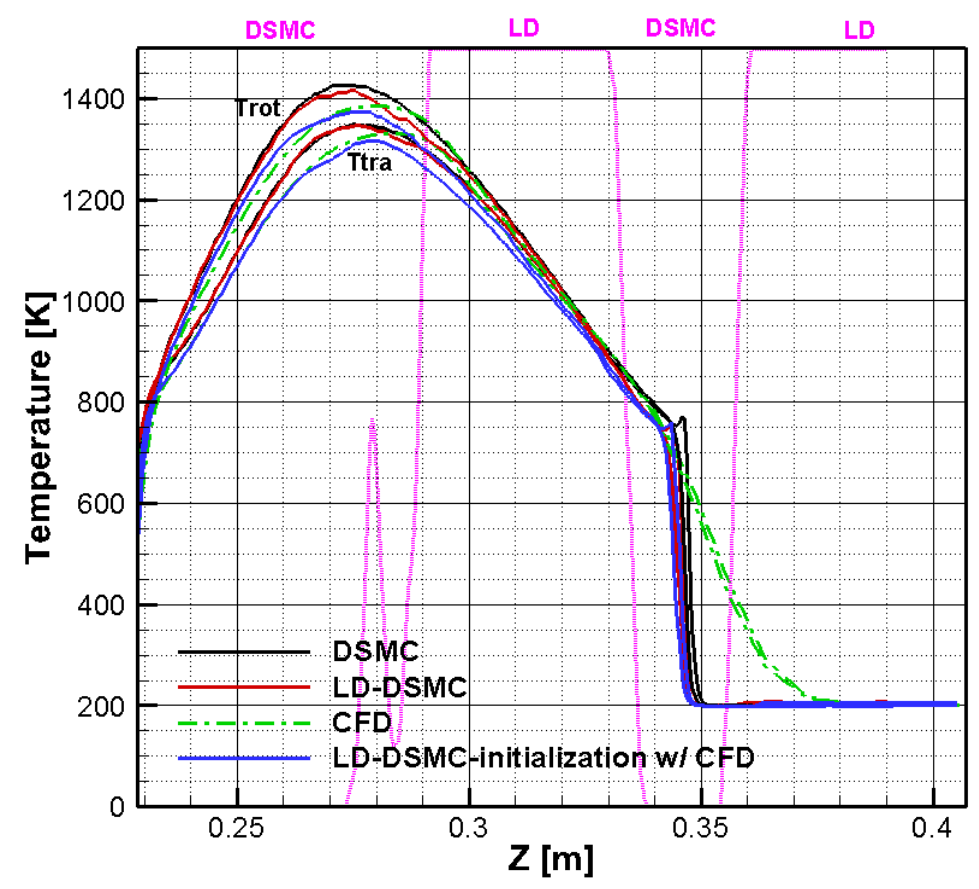

(c) Profiles of temperatures

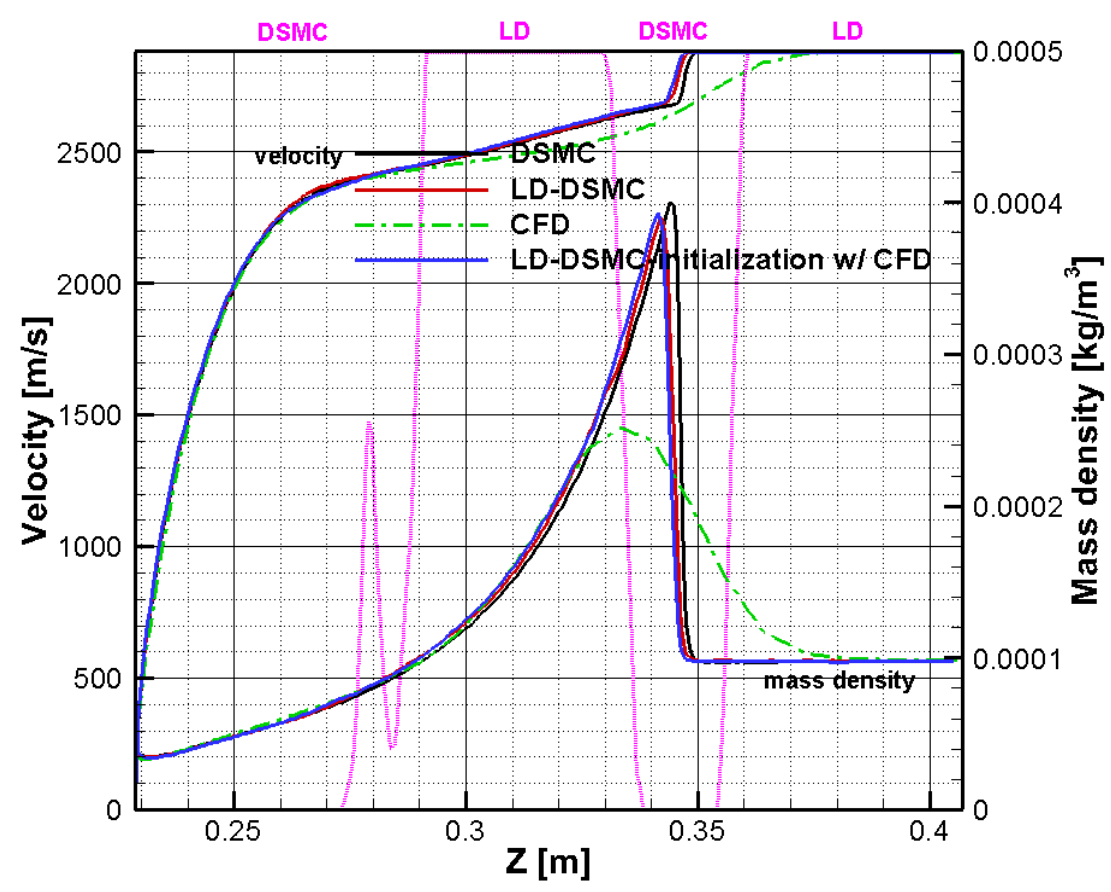

(d) Profiles of bulk velocity and mass density

Figure 15. Profiles along an extraction line inclined at $120^{\circ}$ from the freestream direction: Black and green lines represent standard DSMC and the conventional LD-DSMC hybrid, respectively. Dotted green lines denote CFD results. Blue lines are the LD-DSMC hybrid simulation initialized with Navier-Stokes solution. 


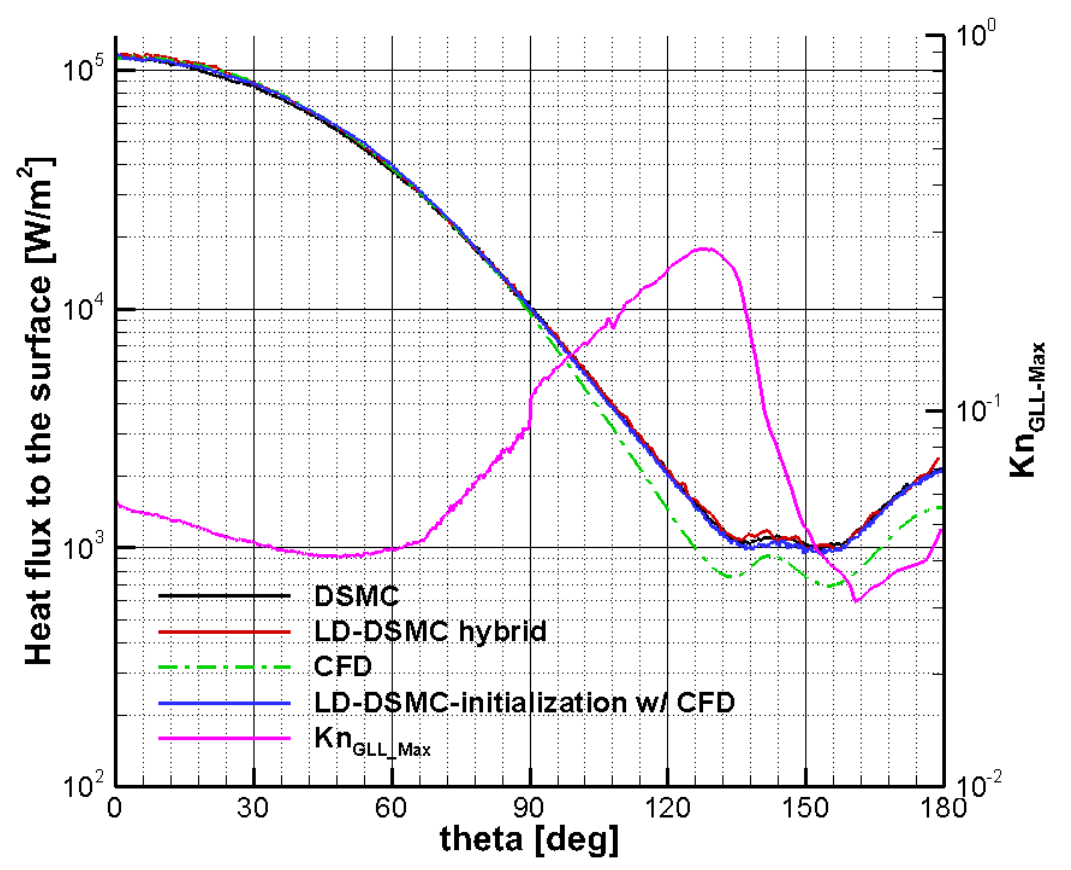

(a) Heat flux to the surface

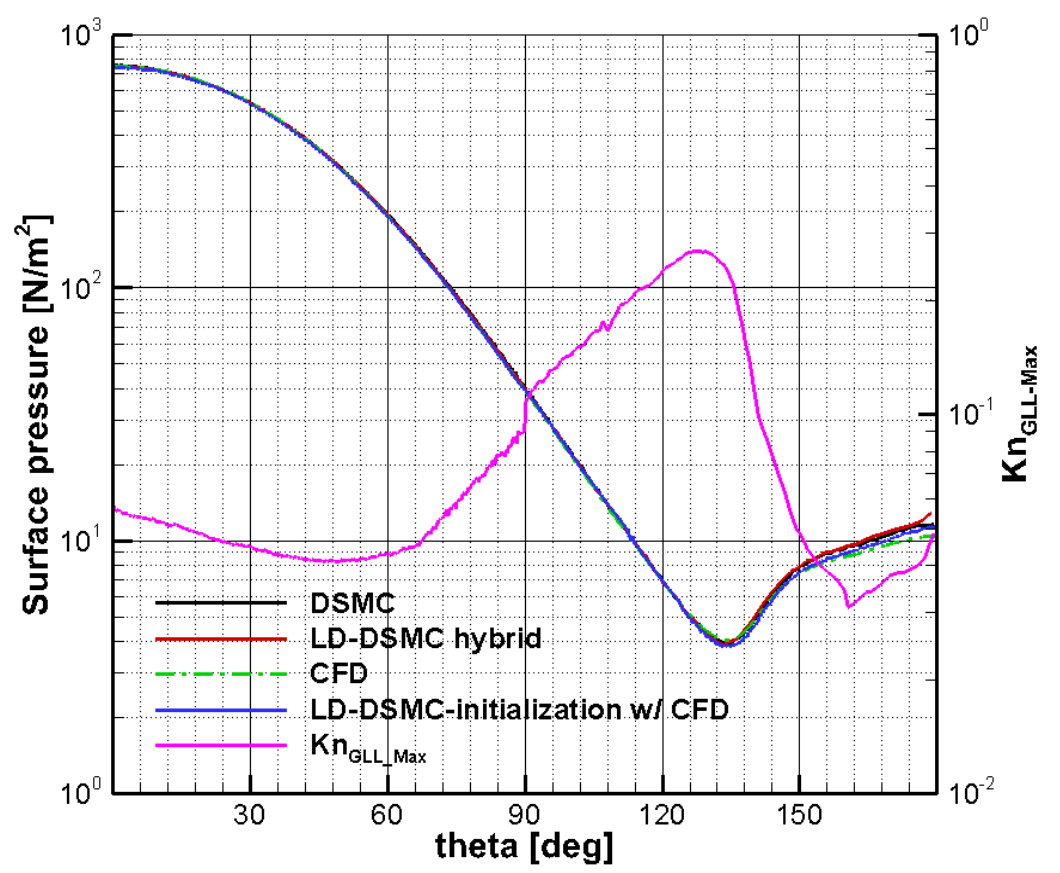

(b) Surface pressure

Figure 16. Profiles along the sphere surface from DSMC, LD-DSMC, CFD, and LD-DSMC initialized with CFD 
Error comparison with DSMC (Heat flux to the surface)

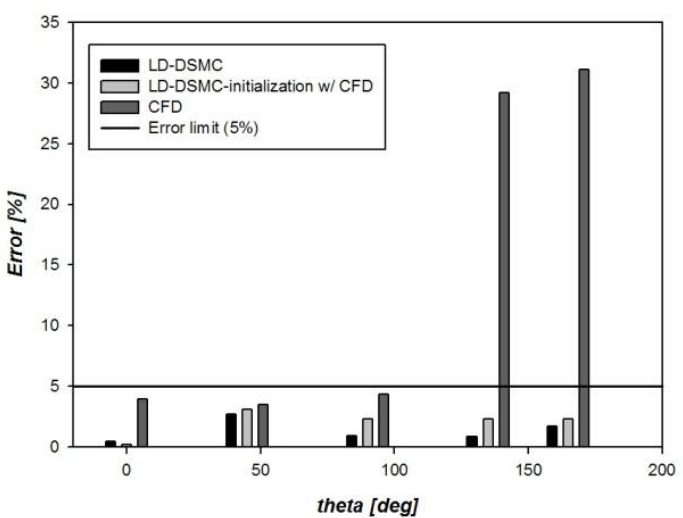

(a) Error comparison of heat flux to the surface
Error comparison with DSMC (Surface pressure)

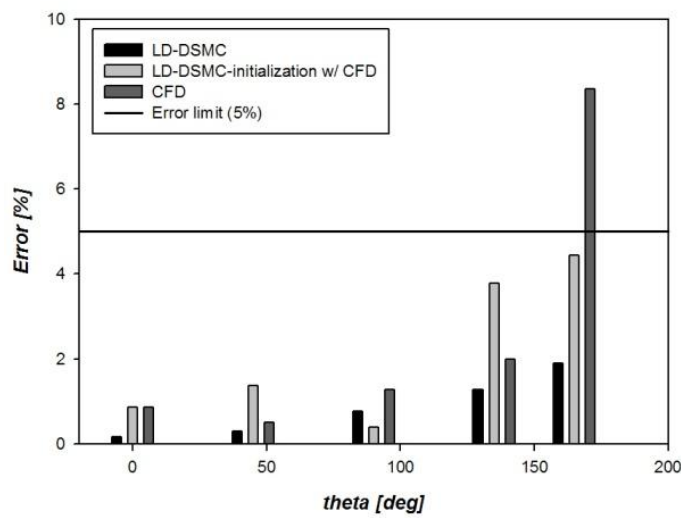

(b) Error comparison of surface pressure

Figure 17. Error comparison of DSMC, LD-DSMC, CFD, and LD-DSMC initialized with CFD for surface properties
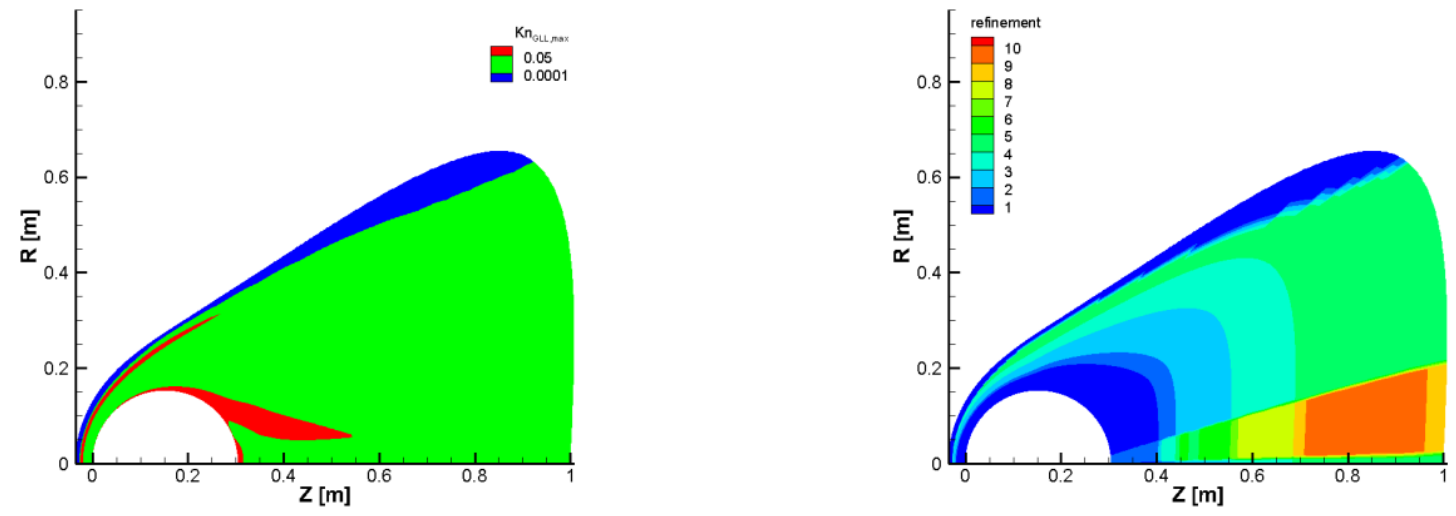

Figure 18. Initial domain decomposition based on Navier-Stokes solution (left) and number of refinement of each CFD cell (right) 

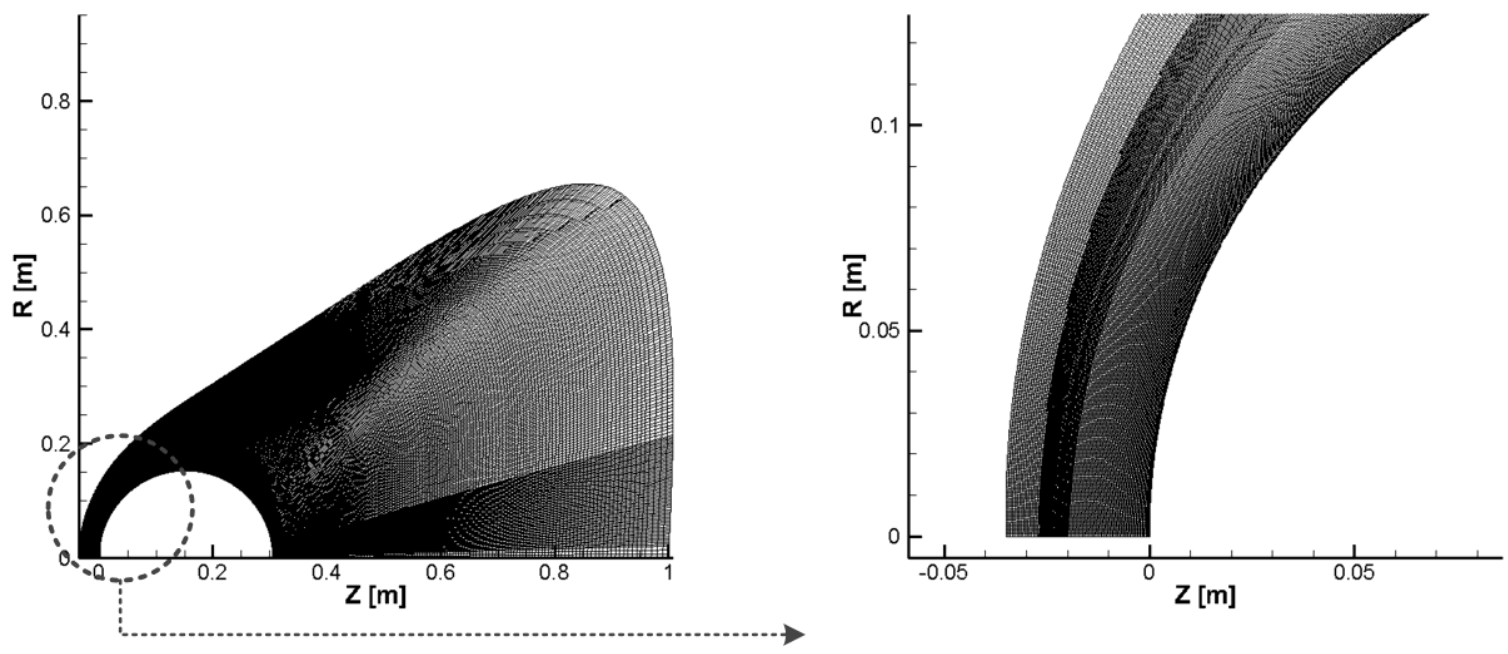

Figure 19. Refined mesh based on Navier-Stokes solution

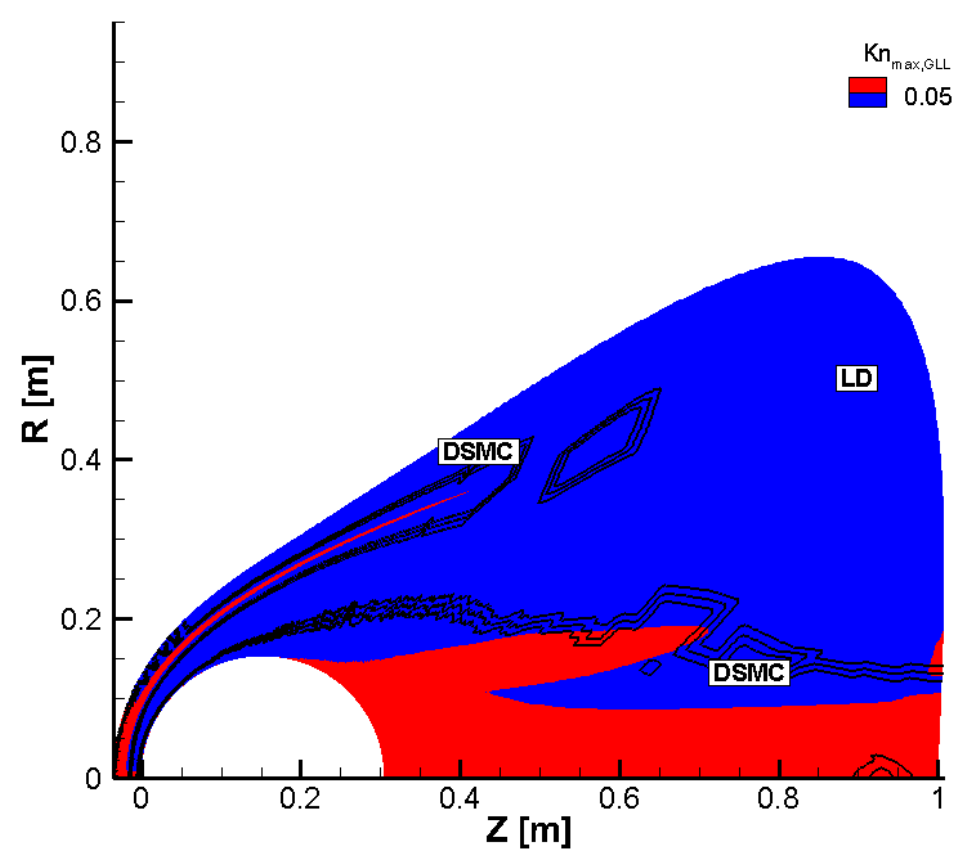

Figure 20. Maximum gradient length local Knudsen number and domain decomposition of LD-DSMC initialized with Navier-Stokes solution 